\title{
A Subband Adaptive Iterative Shrinkage/Thresholding Algorithm
}

\author{
İlker Bayram and Ivan W. Selesnick
}

\begin{abstract}
We investigate a subband adaptive version of the popular iterative shrinkage/thresholding algorithm that takes different update steps and thresholds for each subband. In particular, we provide a condition that ensures convergence and discuss why making the algorithm subband adaptive accelerates the convergence. We also give an algorithm to select appropriate update steps and thresholds for when the distortion operator is linear and time invariant. The results in this paper may be regarded as extensions of the recent work by Vonesch and Unser.
\end{abstract}

Index Terms - Deconvolution, fast algorithm, shrinkage, subband adaptive, thresholding, wavelet regularized inverse problem.

\section{INTRODUCTION}

I $\mathrm{N}$ a typical inverse problem, one is asked to recover an object from distorted and noisy observations of it. Presence of noise and ill-conditioning of the distortion operator lead to an ill-posed problem and render further a priori information about the object necessary [2]. To that end, sparsity of the object in some given (wavelet) frame can be used to regularize the problem. This can be done through a formulation that requires the minimization of a functional composed of a data discrepancy term and a sparsity promoting functional on the frame coefficients. For a particular selection of the functionals, the iterative shrinkage/thresholding algorithm (ISTA) [10] gives a simple method to obtain the minimizer. One iteration of ISTA consists of a Landweber update [2] followed by thresholding the frame coefficients. Despite its simplicity, a disadvantage of ISTA is its slow convergence. In order to accelerate the algorithm, several authors proposed to make the update steps and the thresholds vary for different coefficients [10], [13], [33]. However, save for the Shannon wavelet basis [33], there is not a simple method to select these update steps and thresholds as far as we are aware. In this paper, we address this problem and provide methods to select the update steps and thresholds for different subbands of an arbitrary iterated filter bank (or discrete wavelet transform). We also make precise why this modification accelerates the algorithm by an investigation of the convergence rate.

Manuscript received March 31, 2009; accepted September 07, 2009. First published November 06, 2009; current version published February 10, 2010. The associate editor coordinating the review of this manuscript and approving it for publication was Dr. Ut-Va Koc.

I. Bayram was with the Department of Electrical and Computer Engineering, Polytechnic Institute of New York University, Brooklyn, NY 11201 USA. He is now with the Biomedical Imaging Group, Ecole Polytechnique Fédérale de Lausanne (EPFL), CH-1015, Lausanne, Switzerland.

I. W. Selesnick is with the Department of Electrical and Computer Engineering, Polytechnic Institute of New York University, Brooklyn, NY 11201 USA (e-mail: ilker.bayram@epfl.ch; selesi@poly.edu).

Digital Object Identifier 10.1109/TSP.2009.2036064
Let us now expand the discussion above. Suppose there is an object $x$, we would like to acquire but that we observe only a distorted and noisy version of it, namely $y$, given by

$$
y=H x+n
$$

where $H$ denotes the distortion operator and $n$ noise. Let $\left\{f_{j, k}\right\}_{j=1, k \in \mathbb{Z}}^{J}$ be a wavelet frame where $j, k$ denote the scale and position respectively. To be precise, $\left\{f_{j, k}\right\}_{j=1, k \in \mathbb{Z}}^{J}$ are discrete-time functions derived from an iterated filter bank. Also set $T$ to be the synthesis operator for this frame, acting on the frame coefficients $\mathbf{c}=\left\{c_{j, k}\right\}_{j=1, k \in \mathbb{Z}}^{J}$ as $T \mathbf{c}=\sum_{j, k} c_{j, k} f_{j, k}$. In order to obtain an estimate of $x$, wavelet regularization methods usually constrain the estimate $\hat{x}$ to have a sparse representation using such a wavelet frame. This leads to a formulation where one seeks ${ }^{1}$

$$
\hat{\mathbf{c}} \in \underset{\mathbf{c} \in l_{2}(\mathbb{Z})}{\arg \min } \underbrace{\|y-H T \mathbf{c}\|_{2}^{2}+\lambda\|\mathbf{c}\|_{1}}_{\Phi(\mathbf{c})}
$$

and sets $\hat{x}=T \hat{\mathbf{c}}$. Variants of the following algorithm, which we refer to as ISTA, have been proposed [1], [3], [4], [7], [8], [10]-[12], [16], [17], [28], [36], to obtain the solution $\hat{\mathbf{c}}$. The algorithm is also referred to as the thresholded Landweber algorithm [33], since it consists of a Landweber iteration [2] followed by soft thresholding.

Algorithm 1 (ISTA): Suppose we have an initial estimate $\mathbf{c}$ and $\alpha I-T^{*} H^{*} H T$ is a positive operator. Repeat until some convergence criterion is met

1) Update $\mathbf{c}:=\mathbf{c}+(1 / \alpha) T^{*} H^{*}(y-H T \mathbf{c})$.

2) For $j=1,2, \ldots, J$ and $k \in \mathbb{Z}$, update

$$
c_{j, k}:=\operatorname{soft}\left(c_{j, k}, \lambda /(2 \alpha)\right)
$$

where

$$
\operatorname{soft}(z, t)=\operatorname{sgn}(z) \max \{|z|-t, 0\} .
$$

ISTA treats all the subbands in a uniform manner. However, the effect of the blurring operator is usually felt more severely in some of the subbands than others. Therefore, in those subbands where there is a significant suppression, it might be beneficial to take larger steps towards the minimizer so as to accelerate the algorithm [33]. In this paper, we investigate a generalization of ISTA that allows the use of different thresholds and update

\footnotetext{
${ }^{1}$ This formulation may be regarded as a convexified version of a "sparse" regularized formulation (where an $\ell_{0}$ count would replace the $\ell_{1}$ norm). However, it can also be motivated in a Bayesian setting (see [25], for example).
} 
steps for different subbands, thereby yielding a subband adaptive ISTA (that is, SISTA).

In order to state the algorithm, let us introduce the vector $\alpha=$ $\left(\alpha_{1}, \alpha_{2}, \ldots, \alpha_{J}\right)$, and the diagonal operator $\Lambda_{\alpha}$ that multiplies the $j^{\text {th }}$ subband by $\alpha_{j}$ i.e., $\left(\Lambda_{\alpha} \mathbf{c}\right)_{j, k}=\alpha_{j} \mathbf{c}_{j, k}$. We also write $1 / \alpha=\left(\alpha_{1}^{-1}, \alpha_{2}^{-1}, \ldots, \alpha_{J}^{-1}\right)$. With this notation, we have the following.

Algorithm 2 (SISTA): Suppose we have an initial estimate c and $\Lambda_{\alpha}-T^{*} H^{*} H T$ is a positive operator. ${ }^{2}$ Repeat until some convergence criterion is met

1) Update $\mathbf{c}:=\mathbf{c}+\Lambda_{1 / \alpha}\left[T^{*} H^{*}(y-H T \mathbf{c})\right]$.

2) For $j=1,2, \ldots, J$ and $k \in \mathbb{Z}$, update

$$
c_{j, k}:=\operatorname{soft}\left(c_{j, k}, \lambda /\left(2 \alpha_{j}\right)\right) .
$$

Notice that ISTA can be recovered from SISTA if we set all $\alpha_{k}$ 's equal to each other. In this sense SISTA is a generalization of ISTA.

SISTA and the problem of the selection of $\alpha_{j}$ 's was investigated by Vonesch and Unser in [33] for the Shannon wavelet basis. A more general form of the algorithm was also mentioned but not pursued by Daubechies et al. [10] and Elad et al. [13].

As aforementioned, it may be anticipated that taking larger steps would increase the convergence rate. However, in order to ensure convergence, we also would like to make $\Lambda_{\alpha}-T^{*} H^{*} H T$ a positive operator (see the statement of the SISTA and footnote 1). This paper addresses the problem of how to select $\alpha_{i}$ 's. More concretely, we will show that the aforementioned anticipation that favors choosing small $\alpha_{i}$ 's is indeed true, by relating the convergence rate to the spectral radius of $I-\Lambda_{1 / \alpha} T^{*} H^{*} H T$, and we will present a method to select $\alpha_{i}$ 's so that the algorithm is also guaranteed to decrease $\Phi(\cdot)$ [defined in (2)] monotonically and converge.

\section{A. Prior Work}

ISTA was derived by several groups in different frameworks [5]-[8], [10], [16]. In particular, Figueiredo and Nowak [16] propose the functional $\Phi(\cdot)$ as the log-likelihood function for a restoration problem with Gaussian noise and obtain the algorithm based on an EM approach. Combettes and Wajs (see also [7] and [17]) investigate the minimization of functions given as sums of two convex functions. Characterization of the solution as the fixed point of a proximity operator leads to an iterative algorithm that coincides with ISTA for the particular objective function in this paper. Another approach is discussed by Bredies, Lorenz, and Maass in [6], where they interpret the inclusion of a nondifferentiable regularization term as a generalization of a constraint and regard ISTA as a generalized conditional gradient algorithm. We also refer to [5] for a convergence rate analysis of these algorithms.

In this paper, we will follow the derivation of the algorithm in the majorization-minimization (MM) framework, as done by De Mol and Defrise [24] (which is analyzed further by Daubechies et al. [10]). In a nutshell, the idea of MM algorithms is to relax

\footnotetext{
${ }^{2}$ In fact, it was shown by Combettes and Wajs [8] and Hale et al. [17] that if $2 \Lambda_{\alpha}-T^{*} H^{*} H T$ is positive, then convergence is guaranteed. However, in this paper, we will mainly follow the majorization minimization derivation of De Mol and Defrise [24] and Daubechies et al. [10] which demands that $\Lambda_{\alpha}-$ $T^{*} H^{*} H T$ be positive. We return to this issue briefly at the end of Section III.
}

the minimization problem by replacing the objective function with a surrogate function that depends on the current estimate of the minimizer, which also upper bounds the objective function. Minimizing this surrogate function one obtains a better estimate of the minimizer. Iterating the procedure gives the minimizer of the original problem under certain conditions. We will discuss the MM methodology in more detail in Section II.

Modifying ISTA by utilizing subband-adaptive thresholds and update steps (that is, SISTA) was hinted by Daubechies et al. in [10, Remark 2.4] but not pursued. SISTA was investigated by Vonesch and Unser in [33] for when the Shannon wavelet basis is used. In particular, the selection of $\alpha_{j}$ 's were made based on properties specific to the Shannon wavelet basis and are not generally applicable. In a more recent paper [34], the same authors present a slightly different algorithm that allows employing arbitrary wavelet frames. More specifically, the modified algorithm in [34] applies a Landweber step followed by a soft thresholding operation to a single subband using a threshold and update step adapted to that particular subband and then reflects the change to this subband on the remaining subbands. Cycling through the subbands this way, a multilevel subband-adaptive algorithm is obtained to solve (2). Our approach falls somewhere in between these two papers. We use SISTA thereby retaining the IST approach without any modification and provide a method for the selection of $\alpha_{k}$ 's for an arbitrary wavelet frame. Due to treating the subbands separately, the algorithm in [34] can utilize better (that is, larger in this case) update and threshold steps. The advantage of SISTA is that it runs in parallel, updating all of the subbands at the same time which therefore has a lower cost (compared to the algorithm in [34]) for the same number of iterations.

Even though we presented ISTA with soft-thresholding, other types of thresholding may also be applied. For example, Blumensath and Davies [4] study the problem where the $\ell_{1}$ norm in the objective function is replaced by an $\ell_{0}$ count and show that ISTA with hard-thresholding converges to a local minimum. Another interesting extension, closer in spirit to SISTA is the work of Zhang and Kingsbury [37] where they utilize the dualtree complex wavelet transform and an adaptive Bayesian prior along with the bivariate shrinkage rule described in [9].

\section{B. Outline}

SISTA (and, therefore, ISTA) falls in the general category of MM algorithms [15], [19], [21], [22] under certain conditions. In Section II, we review the main idea behind MM algorithms so as to hint why SISTA is expected to converge faster than ISTA. Following this, we present a derivation of the SISTA in Section III within the MM framework. In Section IV we will describe the set of admissible $\alpha$ 's (rendering $\Lambda_{\alpha}-T^{*} H^{*} H T$ positive) for the case of a general linear operator $H$. When $H$ is known to be time-invariant (or space-invariant ${ }^{3}$ ) as well, we further analyze the problem and give an easy-to-implement recipe for the selection of $\alpha_{j}$ 's in Section V. After that, in Section VI, we discuss a generalization of the results of Section II, and relate the convergence rate to the spectral radius of $I-\Lambda_{1 / \alpha} T^{*} H^{*} H T$. We demonstrate the performance of

\footnotetext{
${ }^{3}$ Throughout the paper, we will use "time-invariant" but there are no constraints about dimension.
} 


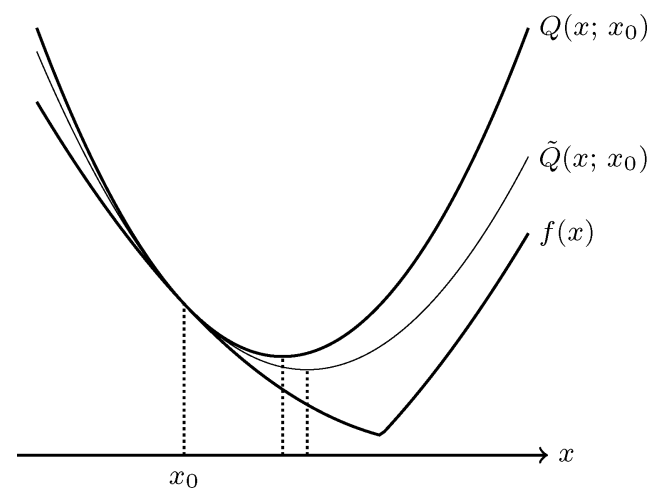

Fig. 1. Two different majorizing functions that touch $f(\cdot)$ at $x_{0}$. Notice that the minimizer of $\tilde{Q}\left(\cdot ; x_{0}\right)$ is closer to the minimizer of $f(\cdot)$.

SISTA in comparison to ISTA and show that further acceleration is also possible by combining SISTA with TwIST (see [3]) in Section VII. Section VIII is the conclusion.

\section{MM ALgORithmS AND RATE OF CONVERGENCE}

For simplicity, consider a 1D convex optimization problem where the task is to find

$$
x^{*}=\arg \min _{x} f(x)
$$

where $f(\cdot): \mathbb{R} \rightarrow \mathbb{R}$ is strictly convex. Also assume that $f(\cdot)$ is complicated enough to preclude any attempt for analytic minimization.

Let $x^{0}$ be a starting point. We wish to find some $x^{1}$ s.t. $f\left(x^{1}\right)<f\left(x^{0}\right)$. Suppose we have a set of functions $\{Q(\cdot ; x)\}_{x \in \mathbb{R}}$ with the properties that

(i) $Q(x ; x)=f(x)$ for all $x \in \mathbb{R}$;

(ii) $Q(y ; x) \geq f(y)$ for all $x, y \in \mathbb{R}$.

Now if we set $x^{1}=\arg \min _{x} Q\left(x ; x^{0}\right)$, (i) and (ii) ensure that $f\left(x^{1}\right) \leq f\left(x^{0}\right)$. Given $x^{0}$ then, the algorithm that sets $x^{k+1}=\arg \min _{x} Q\left(x ; x^{k}\right)$, monotonically decreases the cost $f\left(x_{k}\right)$ with each iteration, converging to $x^{*}$ under mild conditions.

In general, MM does not specify how the majorizing functions $\{Q(\cdot ; x)\}_{x \in \mathbb{R}}$ should be chosen (for some suggestions, see [15] and [19], [22]). However, in order for the algorithm to be practical, $\{Q(\cdot ; x)\}_{x \in \mathbb{R}}$ should be chosen so that they can be minimized (or decreased at least) easily. Still though, there may be many different sets of functions satisfying (i), (ii), which are also easy to minimize. Fig. 1 shows two such candidate functions, $Q\left(x ; x^{0}\right), \tilde{Q}\left(x ; x^{0}\right)$. Notice that $\tilde{Q}\left(x ; x^{0}\right)$ approximates $f(x)$ better than $Q\left(x ; x_{0}\right)$. Intuitively, if $\tilde{Q}(\cdot ; x)$ approximates $f(\cdot)$ better than $Q(\cdot ; x)$ for all $x \in \mathbb{R}$, we expect the iterates $\tilde{x}^{k+1}=\arg \min _{x} \tilde{Q}\left(x ; \tilde{x}^{k}\right)$ to converge faster than $x^{k+1}=\arg \min _{x} Q\left(x ; x^{k}\right)$ for $\tilde{x}^{0}=x^{0}$. Thus, criteria for selecting majorizing functions can be stated as

(a) Ease of minimization;

(b) Good approximation of $f(\cdot)$.

Given the set of functions, $\{Q(\cdot ; x)\}_{x \in \mathbb{R}}$ and a current estimate $x$, one iteration of the $\mathrm{MM}$ algorithm is essentially a mapping $M_{Q}(\cdot): \mathbb{R} \rightarrow \mathbb{R}$, defined by

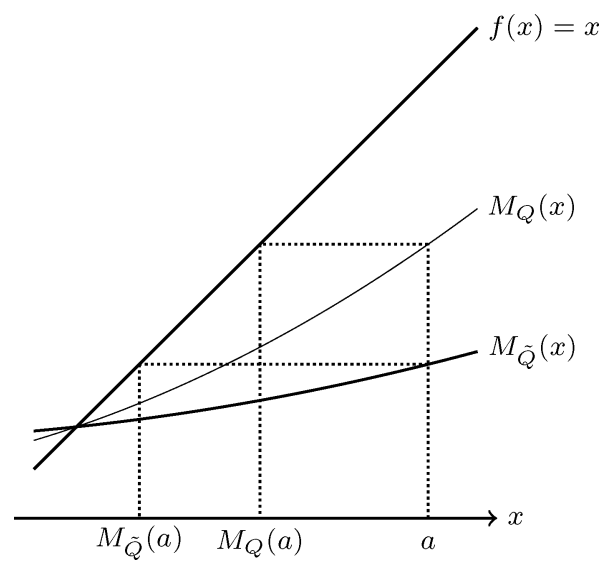

Fig. 2. $M_{Q}(\cdot)$ and $M_{\tilde{Q}}(\cdot)$ have the same fixed point $x^{*}$, but iterating $M_{\tilde{Q}}(\cdot)$ converges to $x^{*}$ faster because $\left|M_{\tilde{Q}}^{\prime}(x)\right|<\left|M_{Q}^{\prime}(x)\right|$ in the vicinity of $x^{*}$.

$M_{Q}(x)=\arg \min _{y \in \mathbb{R}} Q(y ; x)$. Then, convergence of the algorithm to $x^{*}$ is ensured if $x^{*}$ is an attractive fixed point $M_{Q}(\cdot)$. Moreover, the speed of convergence is related to $\left|M_{Q}^{\prime}\left(x^{*}\right)\right|$. Consider Fig. 2 where two mappings $M_{Q}(x)$, $M_{\tilde{Q}}(x)$ associated with the two sets of functions $\{Q(\cdot ; x)\}_{x \in \mathbb{R}}$, $\{\tilde{Q}(\cdot ; x)\}_{x \in \mathbb{R}}$ are shown. They intersect $f(x)=x$ at the same point $x^{*}$, and therefore have the same limit when iterated. However, the figure suggests that starting from the same point, iterations of $M_{\tilde{Q}}(\cdot)$ converge to $x^{*}$ faster because $\left|M_{\tilde{Q}}^{\prime}\left(x^{*}\right)\right|<\left|M_{Q}^{\prime}\left(x^{*}\right)\right|$. Indeed, if we write

$$
M_{Q}(x) \approx M_{Q}\left(x^{*}\right)+M_{Q}^{\prime}\left(x^{*}\right)\left(x-x^{*}\right)
$$

we have

$$
\frac{\left|M_{Q}(x)-x^{*}\right|}{\left|x-x^{*}\right|} \approx\left|M_{Q}^{\prime}\left(x^{*}\right)\right|
$$

since $M_{Q}\left(x^{*}\right)=x^{*}$. Provided $0<\left|M_{Q}^{\prime}\left(x^{*}\right)\right|<1$, it can be shown that, for $x^{k+1}=M_{Q}\left(x^{k}\right)$

$$
\lim _{k \rightarrow \infty}\left|x^{k}-x^{*}\right|^{1 / k}=\left|M_{Q}^{\prime}\left(x^{*}\right)\right| .
$$

Therefore, the magnitude of the derivative of $M_{Q}(\cdot)$ at the limit point determines the convergence rate. In Section VI, we will present a rigorous generalization of this result for our algorithm that is basically an iterated mapping operating on $l_{2}$. The key result will be that the spectral radius of the 'derivative' of this mapping at the fixed point, which in turn will be shown to be bounded by the spectral radius of $I-\Lambda_{1 / \alpha} T^{*} H^{*} H T$, determines the linear convergence rate.

\section{DERIVATION OF SISTA}

Recall that the objective function we are trying to minimize is

$$
\Phi(\mathbf{c})=\|y-H T \mathbf{c}\|_{2}^{2}+\lambda\|\mathbf{c}\|_{1} .
$$

Assuming the frame $\left\{f_{j}, k\right\}_{j=1, k \in \mathbb{Z}}^{J}$ is tight, we can also write

$$
\Phi(\mathbf{c})=\sum_{j=1}^{J} \sum_{k \in \mathbb{Z}} \underbrace{\left|\left\langle y-H T \mathbf{c}, f_{j, k}\right\rangle\right|^{2}}_{\eta_{j, k}}+\lambda\left|c_{j, k}\right| .
$$


The difficulty here is that $\eta_{j, k}$ depends not just on $c_{j, k}$ but several other $c_{n, m}$ 's (with $(n, m) \neq(j, k)$ ). In order to decouple this system of equations, De Mol and Defrise [24] and Daubechies, Defrise, and De Mol [10] add a nonnegative convex functional, $\Xi(\cdot ; \mathbf{a})$ to $\Phi(\cdot)$. These functionals enjoy the property $\Xi(\mathbf{a} ; \mathbf{a})=0$ for all $\mathbf{a}$, thereby yielding an MM type of algorithm, since

(i) $Q(\cdot ; \mathbf{a})=\Xi(\cdot ; \mathbf{a})+\Phi(\cdot) \geq \Phi(\cdot)$;

(ii) $Q(\mathbf{a} ; \mathbf{a})=\Xi(\mathbf{a} ; \mathbf{a})+\Phi(\mathbf{a})=\Phi(\mathbf{a})$ for all $\mathbf{a} \in l_{2}$.

In contrast to $\Xi(\cdot ; \mathbf{a})$, we will use a subband dependent convex positive functional, $\Theta(\cdot ; \mathbf{a})$, leading to a subband dependent version of IST algorithm (see also [10, Remark 2.4]).

\section{A. A Subband-Dependent Surrogate Functional}

First, notice that if $A$ is a positive operator, then $\langle A \mathbf{c}, \mathbf{c}\rangle$ is a convex function of $\mathbf{c}$. Therefore, if $\Lambda_{\alpha}-T^{*} H^{*} H T$ is a positive operator, then

$$
\Theta(\cdot ; \mathbf{a})=\left\langle\Lambda_{\alpha}(\cdot-\mathbf{a}), \cdot-\mathbf{a}\right\rangle-\|H T(\cdot-\mathbf{a})\|_{2}^{2}
$$

is a nonnegative convex function for fixed a with $\Theta(\mathbf{a} ; \mathbf{a})=0$ (compare to $[10, \Xi(f, a)]$ ). Adding this to $\Phi(\cdot)$, we obtain the convex surrogate functional

$$
\begin{gathered}
Q(\mathbf{c} ; \mathbf{a})=\Phi(\mathbf{c})+\Theta(\mathbf{c} ; \mathbf{a}) \\
=\sum_{j=1}^{J} \sum_{k \in \mathbb{Z}}\left\{\alpha_{k}\left|c_{k, i}\right|^{2}-2 c_{j, k}\left[\left(T^{*} H^{*} y\right)_{j, k}+\alpha_{k} a_{j, k}\right.\right. \\
\left.\quad-\left(T^{*} H^{*} H T \mathbf{a}\right)_{j, k}\right] \\
\left.+\lambda\left|c_{k, i}\right|\right\}+g(y ; \mathbf{a})
\end{gathered}
$$

where $g(y ; \mathbf{a})$ is independent of $\mathbf{c}$. Notice that $Q(\mathbf{c} ; \mathbf{a})$ can be minimized (over $\mathbf{c}$ ) by minimizing the function inside the curly brackets for each $\{j, k\}$ pair. For fixed $y$ and $\mathbf{a}$, the function inside the curly brackets is minimized if

$$
x=\operatorname{soft}\left(d_{j, k}, \lambda /\left(2 \alpha_{j}\right)\right)
$$

where

$$
d_{j, k}=\frac{1}{\alpha_{j}}\left(T^{*} H^{*}(y-H T \mathbf{a})\right)_{k, i}+a_{j, k} .
$$

This is a direct consequence of the following well-known result.

Proposition 1: $f(x)=x^{2}-2 z x-\lambda|x|$ attains its minimum value at $x=\operatorname{soft}(z, \lambda / 2)$.

Suppose now that we have an approximate minimizer $\tilde{\mathbf{c}}$ of $\Phi(\cdot)$. Then, since $\Theta(\mathbf{a} ; \mathbf{a})=0$, for all $\mathbf{a} \in l_{2}(\mathbb{Z})$, we get that $Q(\tilde{\mathbf{c}} ; \tilde{\mathbf{c}})=\Phi(\tilde{\mathbf{c}})$. It follows that $\min _{\mathbf{c}} Q(\mathbf{c} ; \tilde{\mathbf{a}}) \leq \Phi(\tilde{\mathbf{c}})$. This leads to SISTA presented in Section I.

A few words about convergence is in order. If the objective function were strictly convex (which would be the case if the operator $H T$ had a trivial null space) the minimizer would be unique. In this case, for $\mathbb{R}^{n}$, convergence of the algorithm to this minimizer follows by noting that the algorithm defines a monotonically descending sequence on $\Phi(\cdot)$ and the convexity of $\Phi(\cdot)$ (see [23, Global Convergence Theorem]). For $l_{2}$, and the case where the objective function is not strictly convex, the situation is more complicated. In [10], Daubechies et al. show that ISTA for $l_{2}$ is convergent by studying the associated operator (iteration of which gives the algorithm). Even though the operator associated with SISTA is different, the analysis in [10] can be adapted and it follows that ISTA for $l_{2}$ is also convergent. Therefore, we can safely assume that " $a$ " minimizer can be obtained by SISTA regardless of the starting point. Last, the papers by Combettes et al. [8] and Hale et al. [17] suggest that SISTA is convergent even if $2 \Lambda_{\alpha}-T^{*} H^{*} H T$ is positive (i.e., $\alpha_{j}$ 's can actually be half of what we proposed). However, if $\Lambda_{\alpha}-T^{*} H^{*} H T$ is not nonnegative, the MM interpretation and monotone decrease of the objective function cease to be true. To gain some insight on this case, let us investigate convergence for the following simplified version of the problem. Suppose that the objective function is strictly convex, that for diagonal $\Lambda$, whose diagonal entries are determined by the vector $\alpha$, we have $(2-2 \epsilon) \Lambda \geq(2-\epsilon) \Lambda-T^{*} H^{*} H T \geq 0$ for some $\epsilon>0$ and that we are in the finite dimensional setting. Then, if we can show that SISTA is a contraction and that the minimizer is a fixed point, ${ }^{4}$ we are done. First, notice that soft thresholding is nonexpansive, i.e., $\|\operatorname{soft}(\mathbf{c})-\operatorname{soft}(\overline{\mathbf{c}})\|_{2} \leq\|\mathbf{c}-\overline{\mathbf{c}}\|_{2}$ and that this is true even if we define a new norm as, $\|\cdot\|_{\Lambda}=\|\Lambda \cdot\|_{2}$, i.e., $\|\operatorname{soft}(\mathbf{c})-\operatorname{soft}(\overline{\mathbf{c}})\|_{\Lambda} \leq\|\mathbf{c}-\overline{\mathbf{c}}\|_{\Lambda}$ (recall that $\Lambda$ is diagonal and invertible). Now consider the first step of SISTA. We want to show that it is a contraction. The first step (i.e., Step 1 in Alg. 2 ) is essentially a mapping defined as

$$
K(\cdot)=\cdot+\Lambda^{-1} T^{*} H^{*}(y-H T \cdot) .
$$

Noting that $(1-\epsilon) \Lambda \geq T^{*} H^{*} H T-\Lambda \geq-(1-\epsilon) \Lambda$, we can write

$$
\begin{aligned}
\|K(\mathbf{c})-K(\overline{\mathbf{c}})\|_{\Lambda} & =\left\|\left(I-\Lambda^{-1} T^{*} H^{*} H T\right)(\mathbf{c}-\overline{\mathbf{c}})\right\|_{\Lambda} \\
& =\left\|\left(\Lambda-T^{*} H^{*} H T\right)(\mathbf{c}-\overline{\mathbf{c}})\right\|_{2} \\
& \leq(1-\epsilon)\|\Lambda(\mathbf{c}-\overline{\mathbf{c}})\|_{2} \\
& =(1-\epsilon)\|(\mathbf{c}-\overline{\mathbf{c}})\|_{\Lambda}
\end{aligned}
$$

Thus, it follows that each iteration of SISTA is a contraction in the modified norm $\|\cdot\|_{\Lambda}$. To see that the minimizer is the unique fixed point of this operator, we can appeal to convex analysis, following the operator splitting approach (see, for example, [7], [8], and [17]), but we can also use the fact that SISTA with $2 \Lambda$ is a convergent MM algorithm and therefore its fixed point is the unique minimizer. In this case, if we denote the minimizer as $\mathbf{c}^{*}$, we have, by the fixed point property [recall (5)]

$$
c_{j, k}=\operatorname{soft}\left(c_{j, k}+b_{j, k} /\left(2 \alpha_{j}\right), \lambda /\left(2 \alpha_{j}\right)\right)
$$

where

$$
\mathbf{b}=T^{*} H^{*}\left(y-H T \mathbf{c}^{*}\right) .
$$

But (21) is equivalent to

$$
\begin{cases}b_{j, k} /\left(2 \alpha_{j}\right)=\operatorname{sgn}\left(c_{j, k}^{*}\right) \lambda /\left(4 \alpha_{j}\right) & \text { if } c_{j, k}^{*} \neq 0 \\ \left|b_{j, k} /\left(2 \alpha_{j}\right)\right|<\lambda /\left(4 \alpha_{j}\right) & \text { if } c_{j, k}^{*}=0\end{cases}
$$

which is equivalent to

$$
\begin{cases}b_{j, k}=\operatorname{sgn}\left(c_{j, k}^{*}\right) \lambda / 2 & \text { if } c_{j, k}^{*} \neq 0 \\ \left|b_{j, k}\right|<\lambda / 2 & \text { if } c_{j, k}^{*}=0\end{cases}
$$

${ }^{4}$ In fact, it is the unique fixed point in this case, by the Banach fixed point theorem [20]. 
It follows by these equivalences that

$$
c_{j, k}=\operatorname{soft}\left(c_{j, k}+b_{j, k} /\left(2 \tilde{\alpha_{j}}\right), \lambda /\left(2 \tilde{\alpha_{j}}\right)\right)
$$

for an arbitrary $\tilde{\alpha}$. In words, $\mathbf{c}^{*}$ is a fixed point of SISTA for any diagonal $\tilde{\Lambda}$, and convergence therefore follows.

In Section IV, we will discuss the problem of selecting $\alpha_{j}$ 's for a general distortion operator $H$. In Section $\mathrm{V}$, we propose a simple recipe for choosing $\alpha_{j}$ 's when $H$ is LTI.

\section{Selection OF $\alpha_{j}$ FOR A Linear Distortion Operator}

Let us partition the synthesis operator $T$ and the frame coefficients into $J$ parts and write

$$
\begin{aligned}
T & =\left[T_{1} T_{2} \ldots T_{J}\right] \\
\mathbf{c} & =\left[\mathbf{c}_{1} \mathbf{c}_{2} \ldots \mathbf{c}_{J}\right]^{T} .
\end{aligned}
$$

Here, $T_{k}$ maps the " $k$ th subband" frame coefficients $\mathbf{c}_{k}$ to the space where $x$ and $y$ from (1) reside in. Now if we define,

$$
S_{j, n}=T_{j}^{*} H^{*} H T_{n}
$$

we can express the operator $T^{*} H^{*} H T$ as,

$$
T^{*} H^{*} H T=\left[\begin{array}{cccc}
S_{1,1} & S_{1,2} & \ldots & S_{1, J} \\
S_{2,1} & \ddots & & S_{2, J} \\
\vdots & & & \vdots \\
S_{J, 1} & \ldots & & S_{J, J}
\end{array}\right]
$$

Notice that for $\hat{\mathbf{c}}=T^{*} H^{*} H T \mathbf{c}$ we have

$$
\hat{\mathbf{c}}_{j}=\sum_{n=1}^{J} S_{j, n} \mathbf{c}_{n} .
$$

By the triangle inequality, we can write

$$
\left\|\hat{\mathbf{c}}_{j}\right\| \leq \sum_{n=1}^{J}\left\|S_{j, n}\right\|\left\|\mathbf{c}_{n}\right\|_{2}
$$

where

$$
\left\|S_{j, n}\right\|=\sup _{\left\|c_{n}\right\|_{2}=1}\left\|S_{j, n}, \mathbf{c}_{n}\right\|_{2}
$$

Therefore

$$
\begin{aligned}
\left\langle T^{*} H^{T} H T \mathbf{c}, \mathbf{c}\right\rangle & =\langle\hat{\mathbf{c}}, \mathbf{c}\rangle \\
& =\sum_{j=1}^{J}\left\langle\hat{\mathbf{c}}_{j}, \mathbf{c}_{j}\right\rangle \\
& \leq \sum_{j=1}^{J}\left\|\hat{\mathbf{c}}_{j}\right\|_{2}\left\|\mathbf{c}_{j}\right\|_{2} \\
& \leq \sum_{j=1}^{J} \sum_{n=1}^{J}\left\|S_{j, n}\right\|\left\|\mathbf{c}_{n}\right\|_{2}\left\|\mathbf{c}_{j}\right\|_{2}
\end{aligned}
$$

where we used (31) in the last line. Now if we define the Hermitian matrix $\mathbf{B}$ as

$$
\mathbf{B}=\left[\begin{array}{cccc}
\left\|S_{1,1}\right\| & \left\|S_{1,2}\right\| & \cdots & \left\|S_{1, J}\right\| \\
\left\|S_{2,1}\right\| & \left\|S_{2,2}\right\| & & \\
\vdots & & \ddots & \\
\left\|S_{J, 1}\right\| & & & \left\|S_{J, J}\right\|
\end{array}\right]
$$

we can express (36) as

$$
\left\langle T^{*} H^{T} H T \mathbf{c}, \mathbf{c}\right\rangle \leq\left[\begin{array}{c}
\left\|\mathbf{c}_{1}\right\|_{2} \\
\left\|\mathbf{c}_{2}\right\|_{2} \\
\vdots \\
\left\|\mathbf{c}_{J}\right\|_{2}
\end{array}\right]^{T} \mathbf{B}\left[\begin{array}{c}
\left\|\mathbf{c}_{1}\right\|_{2} \\
\left\|\mathbf{c}_{2}\right\|_{2} \\
\vdots \\
\left\|\mathbf{c}_{J}\right\|_{2}
\end{array}\right]
$$

Noting also that

$$
\left\langle\Lambda_{\alpha} \mathbf{c}, \mathbf{c}\right\rangle=\left[\begin{array}{c}
\left\|\mathbf{c}_{1}\right\|_{2} \\
\left\|\mathbf{c}_{2}\right\|_{2} \\
\vdots \\
\left\|\mathbf{c}_{J}\right\|_{2}
\end{array}\right]^{T}\left[\begin{array}{cccc}
\alpha_{1} & 0 & \ldots & 0 \\
0 & \alpha_{2} & & \\
\vdots & & \ddots & \\
0 & & & \alpha_{J}
\end{array}\right]\left[\begin{array}{c}
\left\|\mathbf{c}_{1}\right\|_{2} \\
\left\|\mathbf{c}_{2}\right\|_{2} \\
\vdots \\
\left\|\mathbf{c}_{J}\right\|_{2}
\end{array}\right]
$$

we conclude that if $\operatorname{diag}\left(\alpha_{1}, \alpha_{2}, \ldots, \alpha_{J}\right)-\mathbf{B}$ is a positive matrix, then $\Lambda_{\alpha}-T^{*} H^{*} H T$ is a positive operator. The following proposition is useful in this regard.

Proposition 2: Let $\mathbf{B}$ be a Hermitian matrix, whose entries are denoted by $b_{i, j}$. In order for $\operatorname{diag}\left\{\alpha_{1}, \alpha_{2}, \ldots, \alpha_{J}\right\}-\mathbf{B}$ to be positive:

(i) It is necessary that $\alpha_{i}>b_{i i}$ for $i=1,2, \ldots, J$.

(ii) It is sufficient that $\alpha_{i}>\sum_{j=1}^{J}\left|b_{i, j}\right|$.

Proof: These are corollaries of well known results. (i) follows from the fact that the diagonal entries of a Hermitian matrix majorizes its eigenvalues (see [18, Thm. 4.3.26]). (ii) follows from Gershgorin's theorem (see [18, Thm. 6.1.1]).

As a corollary, we have the following.

Proposition 3: If

$$
\alpha_{j}>\sum_{n=1}^{J}\left\|S_{j, n}\right\|
$$

then $\Lambda_{\alpha}-T^{*} H^{*} H T$ is a positive operator.

We remark that even though (40) is a sufficient condition, part (ii) of Prop. 2 implies that the selected $\alpha_{j}$ 's will not be much larger than necessary if the matrix $\mathbf{B}$ is diagonally dominant. However one should keep in mind that (38) is not an equality either.

In a finite dimensional setting, the system $S_{j, n}$ can be represented by a matrix. In this case, Prop. 3 suggests that we take

$$
\alpha_{j}>\sum_{n=1}^{J} \sqrt{\rho\left(S_{j, n}^{*} S_{j, n}\right)} .
$$

where $\rho\left(S_{j, n}^{*} S_{j, n}\right)$ denotes the spectral radius of $S_{j, n}^{*} S_{j, n}$, which is equal to the largest eigenvalue of the nonnegative matrix $S_{j, n}^{*} S_{j, n}$.

Next, we provide a further analysis for the relatively simple but important case of an LTI distortion operator and a frame derived from a filter bank. 


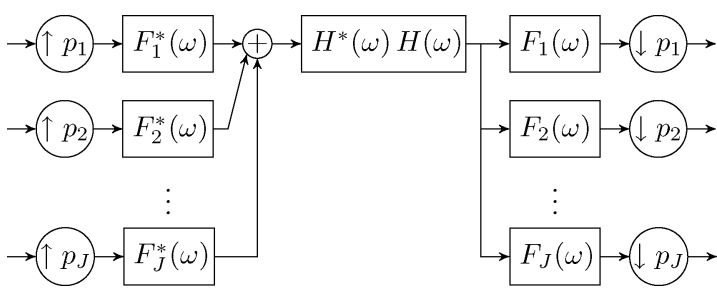

Fig. 3. The operator $T^{*} H^{*} H T$ is realized by the system above. Here, an upsampler by $p_{n}$ inserts $p_{n}-1$ zeros between each sample of its input. A downsample by $p_{n}$ keeps every $k \cdot p_{n}^{\text {th }}$ sample of the input where $k$ ranges over $\mathbb{Z}$. For more details, see [32].

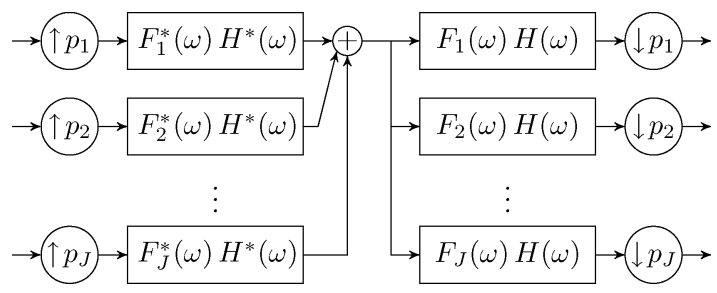

Fig. 4. This system is equivalent to the one in Fig. 3 and realizes the operator $T^{*} H^{*} H T$.

\section{SElection of UPdate STEPs/ThresholdS FOR A DECONVOLUTION PROBLEM}

In this section, we assume that the distortion $H$ is an LTI system and that the frame is derived from a filter bank with $J$ subbands. We will denote the $z$-transform and the DTFT of a filter $f(n)$ as $F(z)$ and $F(\omega)$ respectively. We will assume that all of the filters have DTFTs with at most a finite number of discontinuities, that they have real coefficients and we will set $\tilde{F}(z)=F^{*}\left(z^{-1}\right)$. Other than these, we adopt the notation of the previous sections. We will describe two methods to obtain $\alpha_{j}$ 's s.t. $\Lambda_{\alpha}-T^{*} H^{*} H T$ is positive.

In this setting, since $H$ is an LTI operator, if we denote the filter associated with $H$ by $H(\omega)$, the operator $T^{*} H^{*} H T$ is realized by the system in Fig. 3. In Section V-A, we will transform this system and describe the set of $\alpha$ 's that make $\Lambda_{\alpha}-T^{*} H^{*} H T$ a positive operator. In Section V-B, we will pursue the development in Section IV and describe a selection algorithm for $\alpha$ so that $\Lambda_{\alpha}-T^{*} H^{*} H T$ is positive.

\section{A. Method I: Polyphase Matrix Description of $T^{*} H^{*} H T$}

Notice that, by linearity and associativity, the systems in Fig. 3 and Fig. 4 are equivalent. Now if we set $M=\operatorname{lcm}\left(p_{1}, p_{2}, \ldots, p_{J}\right)$, these systems are also equivalent to a system like the one shown in Fig. 5, where $G_{i}$ 's are determined by $F_{k}(\omega) H(\omega)$ 's and $p_{n}$ 's. Notice that the $k^{\text {th }}$ subband coefficients should also be split into $M / p_{k}$ subbands to accommodate for the transformation. For this $N$-band system, admissible $\alpha_{j}$ 's for $j=1,2, \ldots, N$ can be found using the polyphase description of the system.

For the $i^{\text {th }}$ filter $G_{i}(z)$ the polyphase components $G_{i, k}(z)$ (for $k=0,1, \ldots, N-1$ ) are defined through

$$
G_{i}(z)=\sum_{k=0}^{N-1} z^{-k} G_{i, k}\left(z^{N}\right)
$$

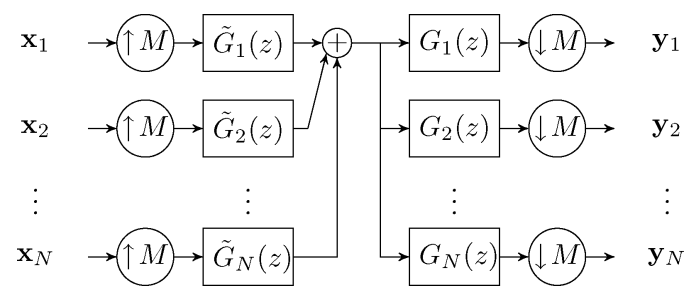

Fig. 5. A uniformly sampled (overcomplete) synthesis and analysis FB in cascade.

Using these, the polyphase matrix $\mathbf{G}(z)$ is constructed by setting the $(i+1, k+1)$ st entry to $G_{i, k}(z)$. It follows, following the notation of Fig. 5, that

$$
\left[\begin{array}{c}
Y_{1}(z) \\
Y_{2}(z) \\
\vdots \\
Y_{N}(z)
\end{array}\right]=\mathbf{G}(z)\left[\begin{array}{c}
X_{1}(z) \\
X_{2}(z) \\
\vdots \\
X_{N}(z)
\end{array}\right] .
$$

If we denote the system in Fig. 5 by $\mathcal{G}$ (this is equivalent to $\left.T^{*} H^{*} H T\right)$, we have the following.

Proposition 4: For $\alpha=\left(\alpha_{1}, \alpha_{2}, \ldots, \alpha_{N}\right), \Lambda_{\alpha}-\mathcal{G}$ is a positive operator if and only if there exists $A>0$ such that $\boldsymbol{\Psi}(\omega)=\operatorname{diag}\left(\alpha_{1}, \alpha_{2}, \ldots, \alpha_{N}\right)-\mathbf{G}\left(e^{j \omega}\right) \mathbf{G}^{*}\left(e^{j \omega}\right)>A I$ almost everywhere.

Proof: Suppose $\boldsymbol{\Psi}(\omega)>A I>0$ almost everywhere. Then

$$
\begin{aligned}
\left\langle\mathbf{x},\left(\Lambda_{\alpha}-\mathcal{G}\right) \mathbf{x}\right\rangle & =\frac{1}{2 \pi} \int_{0}^{2 \pi}\langle X(\omega), \Psi(\omega) X(\omega)\rangle d \omega \\
& \geq \frac{1}{2 \pi} \int_{0}^{2 \pi} A \sum_{i=1}^{N}\left|X_{i}(\omega)\right|^{2} d \omega=A\|x\|^{2} .
\end{aligned}
$$

For the converse, suppose there does not exist such a positive constant $A$. Then, for any given $\epsilon$, we can find a set $\Lambda_{1 / \alpha} \Delta$ with nonzero measure where $\Psi(\omega)<\epsilon I$. Therefore on $\Lambda_{1 / \alpha} \Delta$ we can find $X(\omega)$ with at most a finite number of discontinuities (recall that we assume that the filters have only finitely many discontinuities) such that $\Psi(\omega) X(\omega)=\lambda(\omega) X(\omega)$ with real $\lambda(\omega)<\epsilon$. If we set $X(\omega)$ equal to zero outside of $\Lambda_{1 / \alpha} \Delta$, we get $\left\langle\mathbf{x},\left(\Lambda_{\alpha}-\mathcal{G}\right) \mathbf{x}\right\rangle<\epsilon\|x\|$. Since $\epsilon$ was arbitrary, we have that $\Lambda_{\alpha}-\mathcal{G}$ is not positive and the claim follows.

In principle, since the number of channels is finite, one can determine the family of allowed $\alpha$ 's s.t. $\Lambda_{\alpha}-\mathcal{G}$ is positive. To that end, Prop. 2 may also be utilized, which is likely to give "good" $\alpha_{j}$ 's when $\mathbf{G}\left(e^{j \omega}\right) \mathbf{G}^{*}\left(e^{j \omega}\right)$ is diagonally dominant.

In order to apply Prop. 4 to our original system in Fig. 3, we need to transform the system so that all of the upsampling/downsampling operations are performed with respect to the same integer. One drawback is that the number of subbands can be substantially higher than the number of subbands we originally started with. Our next method pursues the approach presented in Section IV and provides a recipe for the selection of $\alpha_{j}$ 's, without transforming the FB structure. 


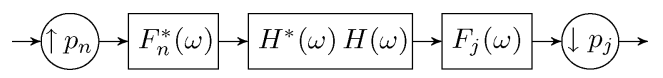

Fig. 6. A component of the system realizing $T^{*} H^{*} H T$. We refer to this system as $S_{j, n}$.

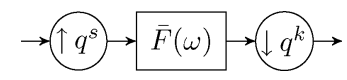

Fig. 7. Provided the FB in Fig. 6 is a DWT type FB (obtained by iterating an FB on its lowpass filter), it is of the form above, i.e. the upsampler and the downsampler is a power of an integer.

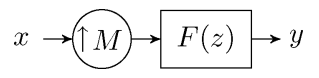

Fig. 8. Norm of this operator is given in Lemma 1.

\section{B. Method II: Decomposition of $T^{*} H^{*} H T$ Into Subsystems}

Recall that Prop. 3 already provides a method to select $\alpha_{j}$ 's if we know $\left\|S_{j, n}\right\|$ 's. For a general linear distortion operator $H$, one has to compute (or at least upper bound) $\left\|S_{j, n}\right\|$ explicitly for all $j \leq n, n=1,2, \ldots, J$. In fact, for a DWT type filter bank (obtained by iterating a multichannel FB), when $H$ is an LTI system, as we assumed in this section, we can express $\left\|S_{j, n}\right\|$ in terms of $H(\omega)$ and $F_{j}(\omega)$.

First notice that following the notation of Fig. 3, the system $S_{j, n}$ can be drawn as in Fig. 6. If the frame is obtained by iterating a filter bank, the system in Fig. 6 can be redrawn as in Fig. 7, where $\bar{F}(\omega)=F_{n}^{*}(\omega) H^{*}(\omega) H(\omega) F_{j}(\omega)$.

Proposition 5: For $S_{k, s}$ given in Fig. 7, set $m=|k-s|$, $l=\min (k, s)$. Also downsample $\bar{F}(z)$ by $q^{l}$ and denote the resulting filter by $\bar{F}_{0}(z)$. Then

$$
\left\|S_{k, s}\right\|^{2}=q^{-m} \operatorname{esssup}_{\omega \in\left[0,2 q^{-m} \pi\right]} \sum_{r=0}^{q^{m}-1}\left|\bar{F}_{0}\left(\omega+r q^{-m} 2 \pi\right)\right|^{2} .
$$

For the proof, we will use the following lemma.

Lemma 1: Let us denote the operator in Fig. 8 by $S$. Then

$$
\|S\|^{2}=\operatorname{ess}_{\omega \in[0,2 \pi / M]} \frac{1}{M} \sum_{r=0}^{M-1}\left|F\left(\omega+r \frac{2 \pi}{M}\right)\right|^{2} .
$$

Proof: Notice $Y(\omega)=F(\omega) X(M \omega)$. Suppose the RHS of (47) evaluates to $B$. Then

$$
\begin{aligned}
\|y\|_{2}^{2} & =\frac{1}{2 \pi} \int_{0}^{2 \pi}|F(\omega) X(M \omega)|^{2} d \omega \\
& =\frac{1}{2 \pi} \int_{0}^{2 \pi / M}\left(\sum_{r=0}^{M-1}\left|F\left(\omega+r \frac{2 \pi}{M}\right)\right|^{2}\right)|X(M \omega)|^{2} d \omega \\
& \leq B \frac{1}{2 \pi} \int_{0}^{2 \pi}|X(\omega)|^{2} d \omega=B\|x\|_{2}^{2}
\end{aligned}
$$

Thus, $\|S\|^{2} \leq B$.

For the converse, given an arbitrary $\epsilon>0$, we can find a set $\Lambda_{1 / \alpha}$ elta $\in[0,2 \pi / M]$ with a nonzero measure s.t. the RHS of (47) is greater than $B-\epsilon$. Now take $X(M \omega)=1$ on $\Lambda_{1 / \alpha}$ elta and zero elsewhere for $\omega \in[0,2 \pi / M]$. Then, it follows by

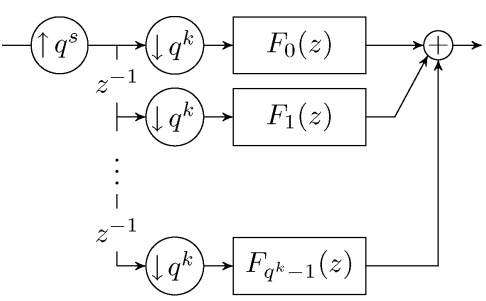

(a)

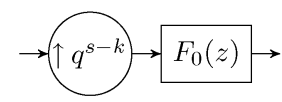

(b)

Fig. 9. For $s \geq k$, it follows by the noble identities that the system in Fig. 7 and the system in (a) are equivalent. Again provided $s \geq k$, the system in (b) is equivalent to (a) since all of the channels except the uppermost one yield zero output.

the train of equalities in (48) that $\|S x\|_{2}^{2} \geq(B-\epsilon)\|x\|_{2}^{2}$. By the arbitrariness of $\epsilon$ then, we have $\|S\|^{2} \geq B$ and the claim follows.

Proof of Prop. 5: First notice that the cases $s>k$ and $k>s$ give transpose system pairs, so their norms are the same. Thus, without loss of generality, we take $s \geq k$.

Now if we denote the $q^{k}$ polyphase components of $F(z)$ by $F_{i}(z)$ for $i=0,1, \ldots, q^{k}-1$, equivalence of the systems in Fig. 7 and Fig. 9(a) follows by the noble identities [32]. That the systems in Fig. 9(a) and Fig. 9(b) are equivalent is also evident by inspection. The proposition now follows by Lemma 1 .

We note that norm analyses of more general multirate systems, where $p_{j}$ 's are not powers of an integer, were provided in the papers by Shenoy [30], Weisburn et al. [35] and Shenroy et al. [31], but the particular case we treat here lead to significant simplifications as indicated by the proposition.

To summarize,

Algorithm 3 (Selection of $\alpha_{j}$ 's for a Deconvolution Problem): Suppose $p_{j}=q^{b_{j}}$ with $b_{j}$ an integer for all $j=1,2, \ldots, J$.

(i) Repeat for $j \leq n, n=1,2, \ldots, J$,

- Set $\bar{F}(z)=\tilde{F}_{n}(z) \tilde{H}(z) H(z) F_{j}(z)$.

- Set $m=\left|b_{j}-b_{n}\right|, l=\min \left(b_{j}, b_{n}\right)$.

- Downsample $\bar{F}(z)$ by $q^{l}$ and denote the resulting filter by $\bar{F}_{0}(z)$.

- Set

$$
\begin{aligned}
& \left\|S_{j, n}\right\|:=q^{-m} \sup _{\omega \in\left[0,2 q^{-m} \pi\right]} \sum_{r=0}^{q^{m}-1}\left|\bar{F}_{0}\left(\omega+r q^{-m} 2 \pi\right)\right|^{2} \\
& \left\|S_{n, j}\right\|:=\left\|S_{j, n}\right\| .
\end{aligned}
$$

(ii) Pick $\alpha_{j}$ s.t. $\alpha_{j}>\sum_{n=1}^{J}\left\|S_{j, n}\right\|$.

We remark that the $\alpha$ vector computed using this algorithm is more effective when the matrix $\mathbf{B}$ [in (38)] is diagonally dominant, which in turn requires that the frequency support of the frame functions employed in different stages do not overlap much. For deconvolution, this means that better frequency selectivity of the frame leads to a tighter upper bound calculation by Algorithm 3. To demonstrate this, we selected as the blurring kernel, the Hamming window of length 5 (in Matlab: 'hamming (5)'). The frequency response of this filter is indicated by the dashed line in Fig. 10(a) and Fig. 10(b). For a 4-stage Haar DWT, using Algorithm 3, we computed the $\alpha$ vector for 


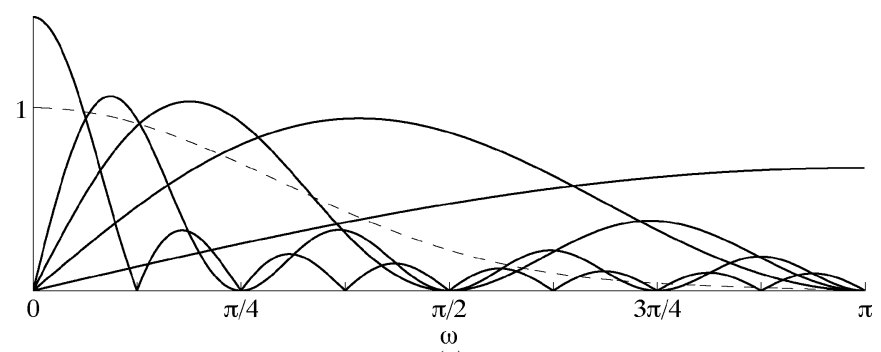

(a)

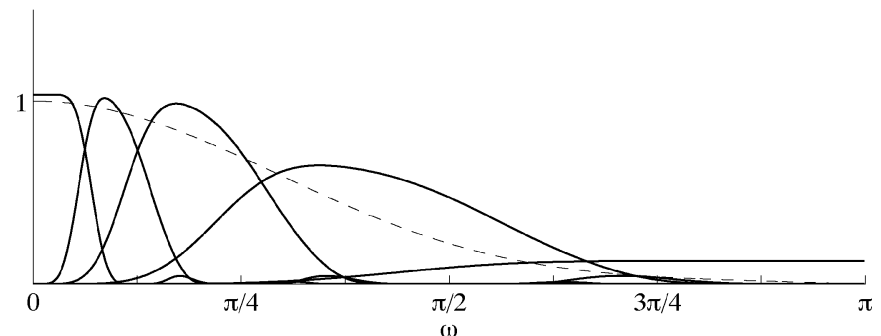

(b)

Fig. 10. The $\alpha$ vector computed using Algorithm 3 improves with the filters' frequency selectivity. In both of the figures, the dashed line depicts the frequency response of the blurring kernel ('hamming (5)'). (a) Frequency response of the Haar DWT weighted by $\alpha$. (b) frequency response of the DWT using Daubechies filters with 6 vanishing moments, weighted by $\alpha$.

this blurring filter and multiplied the (properly normalized) frequency response of each subband with the corresponding entry in $\alpha$. The result is depicted in Fig. 10(a). Notice that there is a significant overshoot in all of the subbands. If we do the same for a DWT using Daubechies filters with 6 vanishing moments, the filters turn out to follow the frequency response of the blurring filter much closely [see Fig. 10(b)]. This requirement of disjoint frequency support may be regarded as a downside of Algorithm 3.

In the next section, we make more precise why the selection of small $\alpha_{j}$ 's speed up the algorithm by generalizing the treatment in Section II.

\section{CONVERgENCE Rate OF SISTA}

In order to draw a parallel with the development and notation of the MM algorithm in Section II, we regard SISTA as a repeated application of a fixed operator, $\mathcal{M}_{\alpha}$, defined by

$$
\mathcal{M}_{\alpha}(\mathbf{c})=\Gamma_{\alpha}\left\{\Lambda_{1 / \alpha}\left[T^{*} H^{*}(y-H T \mathbf{c})\right]+\mathbf{c}\right\}
$$

where $\Gamma_{\alpha}$ is a subband dependent soft-threshold operator with a threshold equal to $\lambda /\left(2 \alpha_{j}\right)$ for the $j^{\text {th }}$ subband. With this definition, for a given starting point $\mathbf{c}$, SISTA is equivalent to repeatedly updating $\mathbf{c}$ as

$$
\mathbf{c}:=\mathcal{M}_{\alpha}(\mathbf{c})
$$

$\mathcal{M}_{\alpha}$ is an operator mapping $l_{2}$ to $l_{2}$. However, we will now show that $\mathcal{M}_{\alpha}$ maps a neighborhood of the minimizer (or its fixed point) into a fixed finite dimensional space determined solely by the minimizer (we also refer to [5] and [17] for detailed analyses). This will allow us to express the convergence rate explicitly in terms of the spectral radius of $I-\Lambda_{1 / \alpha} T^{*} H^{*} H T$.

First notice that, for $\mathbf{c} \in l_{2},(\Gamma(\mathbf{c}))_{j, k}$ will be nonzero for a finite number of $(j, k)$ pairs, otherwise we could find infinitely many $(j, k)$ pairs such that $\left|\mathbf{c}_{j, k}\right| \geq \lambda /\left(2 \alpha_{j}\right)$ and thus $\mathbf{c}$ would not have a finite norm. Noting, for $\mathbf{c} \in l_{2}, \Lambda_{1 / \alpha}\left[T^{*} H^{*}(y-\right.$ $H T \mathbf{c})]+\mathbf{c}$ is also in $l_{2}$ we, therefore, have that $\left(\mathcal{M}_{\alpha}(\mathbf{c})\right)_{j, k}$ can be nonzero only for finitely many $(j, k)$ pairs. Now since the limit point $\mathbf{c}^{*}$ satisfies $\mathbf{c}^{*}=\mathcal{M}_{\alpha}\left(\mathbf{c}^{*}\right)$, it follows that $\mathbf{c}_{j, k}^{*}$ is nonzero for only a finite number of $(j, k)$ pairs.

For convenience of notation, let us denote the operator projecting elements of $l_{2}$ to the support of $\mathbf{c}^{*}$ as $D_{\mathbf{c}^{*}}$

$$
\left(D_{\mathbf{c}^{*}}(\mathbf{c})\right)_{k, i}= \begin{cases}c_{k, i} & \text { if } c_{k, i}^{*} \neq 0 \\ 0 & \text { if } c_{k, i}^{*}=0 .\end{cases}
$$

The following number will be useful for defining a convenient neighborhood around $\mathbf{c}^{*}$.

$$
\epsilon_{\mathbf{c}^{*}}=\inf \left\{\left|\lambda /\left(2 \alpha_{j}\right)-\right| \hat{c}_{j, k}^{*}||: k \in \mathbb{Z}, \quad j \in 1,2, \ldots, J\right\}
$$

where

$$
\hat{\mathbf{c}}^{*}=\Lambda_{1 / \alpha} T^{*} H^{*} y+\left(I-\Lambda_{1 / \alpha} T^{*} H^{*} H T\right) \mathbf{c}^{*} .
$$

We remark that if $\hat{c}_{j, k}^{*} \neq \lambda /\left(2 \alpha_{j}\right)$ for every $(j, k)$ pair, then $\epsilon_{\mathbf{c}^{*}}$ will be a positive number (otherwise $\hat{\mathbf{c}}^{*}$ would not be in $l_{2}$ as argued before).

Lemma 2: Suppose that $\hat{\mathbf{c}}_{j, k}^{*} \neq \lambda /\left(2 \alpha_{j}\right)$ for every $(j, k)$ pair. Set $r=\left\|I-\Lambda_{1 / \alpha} T^{*} H^{*} H T\right\|$. If $\left\|\mathbf{c}-\mathbf{c}^{*}\right\|<\epsilon_{\mathbf{c}^{*}} / r$ then $\left(\mathcal{M}_{\alpha}(\mathbf{c})\right)_{j, k}$ is nonzero if and only if $c_{j, k}^{*}$ is nonzero.

Notice

Proof: Set $\hat{\mathbf{c}}=\Lambda_{1 / \alpha} T^{*} H^{*} y+\left(I-\Lambda_{1 / \alpha} T^{*} H^{*} H T\right) \mathbf{c}$.

$$
\hat{\mathbf{c}}-\hat{\mathbf{c}}^{*}=\left(I-\Lambda_{1 / \alpha} T^{*} H^{*} H T\right)\left(\mathbf{c}-\mathbf{c}^{*}\right) .
$$

Therefore

$$
\left\|\hat{\mathbf{c}}-\hat{\mathbf{c}}^{*}\right\|<\epsilon_{\mathbf{c}^{*}}
$$

Now suppose we can find a $(j, k)$ pair such that $(\Gamma(\hat{\mathbf{c}}))_{j, k} \neq$ 0 but $c_{j, k}^{*}=0$. This implies that $\left|\hat{c}_{j, k}-\hat{c}_{j, k}^{*}\right| \geq \epsilon_{\mathbf{c}^{*}}$. Thus, $\| \hat{\mathbf{c}}-\hat{\mathbf{c}}^{*}|| \geq\left|\hat{c}_{j, k}-\hat{c}_{j, k}^{*}\right| \geq \epsilon_{\mathbf{c}^{*}}$, a contradiction.

By a similar argument, $c_{j, k}^{*} \neq 0$ implies that $(\Gamma(\hat{\mathbf{c}}))_{j, k} \neq 0$ and the lemma follows.

These results, along with the fact that the algorithm converges, implies that $\mathcal{M}_{\alpha}$ becomes essentially a mapping of $\mathbb{R}^{N}$ into $\mathbb{R}^{N}$ where $N$ is the number of $(j, k)$ pairs for which $\mathbf{c}_{j, k}^{*}$ is nonzero (or the dimension of the range of $D_{\mathbf{c}^{*}}$ ). In the following, we will further assume that the minimizer is also unique. ${ }^{5}$ Such iterated mappings are well studied (see, for example, [26]).

In order to generalize the assertions of Section II, let us now make a few definitions and state some results.

Definition 1: We will say that $M: \mathbb{R}^{N} \rightarrow \mathbb{R}^{N}$ defines a convergent algorithm if for any given $\mathbf{a}^{0} \in \mathbb{R}^{N}, \mathbf{a}^{k+1}=M\left(\mathbf{a}^{k}\right)$ converges to the same point $\mathbf{a}^{*}$.

${ }^{5}$ Recall that this is in fact true when $H T$ has a trivial nullspace. However, this might leave out the important case where the underlying frame is overcomplete, so that $T$ has a nontrivial nullspace. Nevertheless, we hope that the analysis that follows sheds some light for such cases as well. 
Given an operator $M$ that defines a convergent algorithm, the question is: How fast is the convergence? We now define a relevant convergence rate.

Definition 2 ([26, Defn. 9.2.1]): Let $\left\{\mathbf{a}^{k}\right\}_{k \in \mathbb{N}}$ be a sequence in $\mathbb{R}^{N}$ (i.e., $\mathbf{a}^{k} \in \mathbb{R}^{N}$ for all $k \in \mathbb{N}$ ) that converges to $\mathbf{a}^{*}$. Set

$$
R\left(\left\{\mathbf{a}^{k}\right\}_{k \in \mathbb{N}}\right)=\limsup _{k \rightarrow \infty}\left\|\mathbf{a}^{k}-\mathbf{a}^{*}\right\|^{1 / k} .
$$

This definition specifies a rate for a single sequence. However, our algorithm produces different sequences for different starting points.

Definition 3(R-Factor): Let $C$ denote the set of all sequences generated by iterating a mapping $M: \mathbb{R}^{N} \rightarrow \mathbb{R}^{N}$. Suppose also that all of these sequences converge to $\mathbf{a}^{*}$. The $R$-factor of $M$ is

$$
\mathcal{R}(M)=\sup \left\{R\left(\left\{x_{k}\right\}_{k \in \mathbb{N}}\right):\left\{x_{k}\right\}_{k \in \mathbb{N}} \in C\right\} .
$$

Recall that for 1D, the magnitude of the derivative of $M$ at the fixed point provided a rate of convergence. For a generalization of this result to our case, we first need to adapt the definition of the derivative.

Definition 4 ([26, Defn. 3.1.5]): A mapping $M: \mathbb{R}^{N} \rightarrow \mathbb{R}^{N}$ is called Frechet (F-) differentiable at $x \in \mathbb{R}^{N}$ if there is a linear mapping $A: \mathbb{R}^{N} \rightarrow \mathbb{R}^{N}$ s.t.

$$
\lim _{h \rightarrow 0} \frac{\|M(x+h)-M(x)-A h\|}{\|h\|}=0 .
$$

In this case, the linear operator $A$ is denoted by $M^{\prime}(x)$ and is called the F-derivative of $M$ at $x$.

We can now state a result analogous to (9).

Proposition 6 ([26, Thm 10.1.4]): Let $M: \mathbb{R}^{N} \rightarrow \mathbb{R}^{N}$ define a convergent algorithm with limit point $\mathbf{a}^{*}$. Also suppose $M^{\prime}\left(\mathbf{a}^{*}\right)$ exists and the spectral radius of $M^{\prime}\left(\mathbf{a}^{*}\right)$ satisfies $0<\rho\left(M^{\prime}\left(\mathbf{a}^{*}\right)\right)<1$. Then, $\mathcal{R}(M)=\rho\left(M^{\prime}\left(\mathbf{a}^{*}\right)\right)$.

Since we know that in the vicinity of the limit $\mathbf{c}^{*}, \mathcal{M}_{\alpha}$ becomes a mapping from a finite dimensional space into itself, the derivative of $\mathcal{M}_{\alpha}$ restricted to this finite dimensional space will determine the $R$-factor for $\mathcal{M}_{\alpha}$.

Proposition 7: Suppose that $\hat{\mathbf{c}}_{j, k}^{*} \neq \lambda /\left(2 \alpha_{j}\right)$ for every $(j, k)$ pair. In this case, the derivative of $\mathcal{M}_{\alpha}\left(\mathbf{c}^{*}\right)$ regarded as a mapping from the range space of $D_{\mathbf{c}^{*}}$ into itself is given by

$$
\mathcal{M}_{\alpha}^{\prime}\left(\mathbf{c}^{*}\right)=D_{\mathbf{c}^{*}}\left(I-\Lambda_{1 / \alpha} T^{*} H^{*} H T\right) .
$$

Proof: Set $r=\left\|I-\Lambda_{1 / \alpha} T^{*} H^{*} H T\right\|$. Pick $h$ in the range space of $D_{\mathbf{c}^{*}}$ with $\|h\|<\epsilon_{\mathbf{c}^{*}} / r$. Let $(j, k)$ be a pair such that $\mathbf{c}_{j, k}^{*} \neq 0$. It follows by Lemma 2 that $\left(\mathcal{M}_{\alpha}\left(\mathbf{c}^{*}+h\right)\right)_{j, k} \neq 0$. For this $(j, k)$ pair

$$
\begin{aligned}
\left(\mathcal{M}_{\alpha}\right. & \left.\left(\mathbf{c}^{*}+h\right)-\mathcal{M}_{\alpha}\left(\mathbf{c}^{*}\right)\right)_{j, k} \\
= & \left(\left(I-\Lambda_{1 / \alpha} T^{*} H^{*} H T\right)\left(\mathbf{c}^{*}+h\right)-\operatorname{sgn}\left(\mathbf{c}_{j, k}^{*}\right) \lambda /\left(2 \alpha_{j}\right)\right)_{j, k} \\
& \quad-\left[\left(\left(I-\Lambda_{1 / \alpha} T^{*} H^{*} H T\right)\left(\mathbf{c}^{*}\right)-\operatorname{sgn}\left(\mathbf{c}_{j, k}^{*}\right) \lambda /\left(2 \alpha_{j}\right)\right)_{j, k}\right] \\
= & \left(\left(I-\Lambda_{1 / \alpha} T^{*} H^{*} H T\right) h\right)_{j, k} .
\end{aligned}
$$

Also, if $c_{j, k}^{*}$ is zero, then both $\left(\mathcal{M}_{\alpha}\left(\mathbf{c}^{*}\right)_{j, k}\right.$ and $\left(\mathcal{M}_{\alpha}\left(\mathbf{c}^{*}+h\right)_{j, k}\right.$ are zero. Therefore

$$
\mathcal{M}_{\alpha}\left(\mathbf{c}^{*}+h\right)-\mathcal{M}_{\alpha}\left(\mathbf{c}^{*}\right)=D_{\mathbf{c}^{*}}\left(I-\Lambda_{1 / \alpha} T^{*} H^{*} H T\right) h .
$$

Since $D_{\mathbf{c}^{*}}\left(I-\Lambda_{1 / \alpha} T^{*} H^{*} H T\right)$ is a linear operator, it must be the $F$-derivative we are looking for.

Corollary 1: The $R$-factor of $\mathcal{M}_{\alpha}$ is equal to the spectral radius of $D_{\mathbf{c}^{*}}\left(I-\Lambda_{1 / \alpha} T^{*} H^{*} H T\right)$.

For linear operators $A, B$, we remark that $\rho(A B) \leq$ $\rho(A) \rho(B)$. Therefore $\alpha$ 's give us some means to accelerate the algorithm by making $\rho\left(I-\alpha_{1 / \alpha} T * H^{*} H T\right)$ as small as possible. To that end, the following proposition provides a guideline.

Proposition 8: Suppose we are given $\alpha=\left(\alpha_{1}, \ldots, \alpha_{J}\right), \tilde{\alpha}=$ $\left(\tilde{\alpha}_{1}, \ldots, \tilde{\alpha}_{J}\right)$ such that $\tilde{\alpha}_{k} \geq \alpha_{k}$, for $k=1, \ldots, J$. If $\Lambda_{\alpha}-$ $T^{*} H^{*} H T$ is a positive operator, then

(i) $\Lambda_{\tilde{\alpha}}-T^{*} H^{*} H T$ is a positive operator.

(ii) $\rho\left(D_{\mathbf{c}^{*}}\left(I-\Lambda_{1 / \tilde{\alpha}} T^{*} H^{*} H T\right)\right) \quad \geq \rho\left(D_{\mathbf{c}^{*}}(I \quad-\right.$ $\left.\left.\Lambda_{1 / \alpha} T^{*} H^{*} H T\right)\right)$.

Proof:

(i) Notice that

$$
\Lambda_{\tilde{\alpha}}-T^{*} H^{*} H T=\Lambda_{\tilde{\alpha}-\alpha}+\left(\Lambda_{1 / \alpha}-T^{*} H^{*} H T\right) \text {. }
$$

Since the sum of a nonnegative and a positive operator is positive, the claim in (i) follows.

(ii) We remark that, in the following, the operators map a fixed finite-dimensional space (namely the range space of $D_{\mathbf{c}^{*}}$ ) to itself and therefore may be taken as matrices.

First, notice that all eigenvalues of $\Lambda_{1 / \alpha} D_{\mathbf{c}^{*}} T^{*} H^{*} H T D_{\mathbf{c}^{*}}$ are positive since it is a product of positive operators. Also, $\lambda$ is an eigenvalue of $\Lambda_{1 / \alpha} D_{\mathbf{c}^{*}} T^{*} H^{*} H T D_{\mathbf{c}^{*}}$ if and only if $1-\lambda$ is an eigenvalue of $D_{\mathbf{c}^{*}}-D_{\mathbf{c}^{*}} \Lambda_{1 / \alpha} T^{*} H^{*} H T D_{\mathbf{c}^{*}}$. Therefore, finding the minimum eigenvalue of $\Lambda_{1 / \alpha} D_{\mathbf{c}^{*}} T^{*} H^{*} H T D_{\mathbf{c}^{*}}$ suffices to compute $\rho\left(D_{\mathbf{c}^{*}}-D_{\mathbf{c}^{*}} \Lambda_{1 / \alpha} T^{*} H^{*} H T D_{\mathbf{c}^{*}}\right)$. The same argument is valid if $\alpha$ is replaced with some $\tilde{\alpha}$ as in the hypothesis.

On the range space of $D_{\mathbf{c}^{*}}$, if $D_{\mathbf{c}^{*}} T^{*} H^{*} H T D_{\mathbf{c}^{*}}$ is not invertible, it has a zero eigenvalue and $\rho\left(D_{\mathbf{c}^{*}}-D_{\mathbf{c}^{*}} \Lambda_{1 / \alpha} T^{*} H^{*} H T D_{\mathbf{c}^{*}}\right)=\rho\left(D_{\mathbf{c}^{*}}\right.$ $\left.D_{\mathbf{c}^{*}} \Lambda_{1 / \tilde{\alpha}} T^{*} H^{*} H T D_{\mathbf{c}^{*}}\right)=1$ and the claim is true trivially.

Now suppose $D_{\mathbf{c}^{*}} T^{*} H^{*} H T D_{\mathbf{c}^{*}}$ is invertible. We remark that if a finite dimensional linear operator $A$ has positive eigenvalues, its minimum eigenvalue is given by $1 / \rho\left(A^{-1}\right)$. Therefore

$$
\begin{aligned}
\rho\left(D_{\mathbf{c}^{*}}-D_{\mathbf{c}^{*}} \Lambda_{1 / \alpha} T^{*} H^{*} H T D_{\mathbf{c}^{*}}\right) & 1 \\
& =1-\frac{1}{\rho\left(\left(D_{\mathbf{c}^{*}} T^{*} H^{*} H T D_{\mathbf{c}^{*}}\right)^{-1} \Lambda_{\alpha}\right)}
\end{aligned}
$$

and

$$
\begin{array}{r}
\rho\left(D_{\mathbf{c}^{*}}-D_{\mathbf{c}^{*}} \Lambda_{1 / \tilde{\alpha}} T^{*} H^{*} H T D_{\mathbf{c}^{*}}\right) \\
\quad=1-\frac{1}{\rho\left(\left(D_{\mathbf{c}^{*}} T^{*} H^{*} H T D_{\mathbf{c}^{*}}\right)^{-1} \Lambda_{\tilde{\alpha}}\right)} .
\end{array}
$$

Now if we denote $\alpha / \tilde{\alpha}=\left(\alpha_{1} / \tilde{\alpha}_{1}, \alpha_{2} / \tilde{\alpha}_{2}, \ldots, \alpha_{J} / \tilde{\alpha}_{J}\right)$, we have

$$
\begin{aligned}
& \rho\left(\left(D_{\mathbf{c}^{*}} T^{*} H^{*} H T D_{\mathbf{c}^{*}}\right)^{-1} \Lambda_{\alpha}\right) \\
& \quad=\rho\left(\left(D_{\mathbf{c}^{*}} T^{*} H^{*} H T D_{\mathbf{c}^{*}}\right)^{-1} \Lambda_{\tilde{\alpha}} \Lambda_{\alpha / \tilde{\alpha}}\right) \\
& \quad \leq \rho\left(\left(D_{\mathbf{c}^{*}} T^{*} H^{*} H T D_{\mathbf{c}^{*}}\right)^{-1} \Lambda_{\tilde{\alpha}}\right) \rho\left(\Lambda_{\alpha / \tilde{\alpha}}\right) \\
& \quad \leq \rho\left(\left(D_{\mathbf{c}^{*}} T^{*} H^{*} H T D_{\mathbf{c}^{*}}\right)^{-1} \Lambda_{\tilde{\alpha}}\right)
\end{aligned}
$$

since $\rho\left(\Lambda_{\alpha / \tilde{\alpha}}\right) \leq 1$. This implies the claim by (64), (65). 


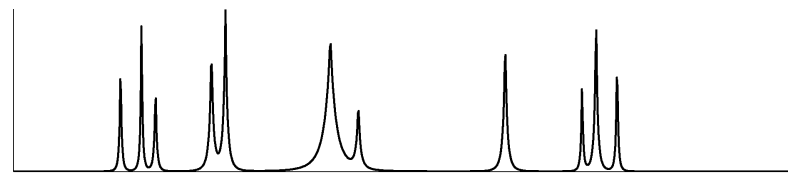

(a)

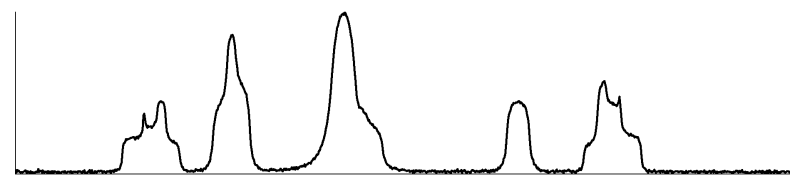

(b)

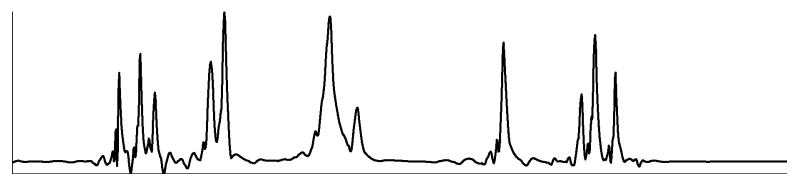

(c)

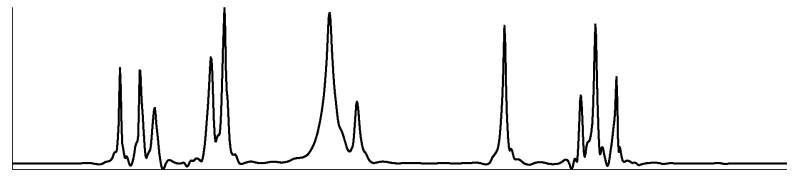

(d)

Fig. 11. (a) Original signal. (b) Observation. (c) Result of ISTA for 50000 iterations with a 6-stage (7-subband) dyadic DWT with Daubechies filters having 4 vanishing moments. (d) Result of ISTA for 50000 iterations using a 4-stage (9-subband) double-density DWT (see [29]).

TABLE I

' $\alpha$ ' VECTOR COMPUTED FOR EXPERIMENT 1. 'LP' STANDS FOR LOWPASS CHANNEL

\begin{tabular}{ccccccc}
\hline 1 & 2 & 3 & 4 & 5 & 6 & 6 (LP) \\
\hline 0.0036 & 0.0121 & 0.0473 & 0.1646 & 0.7155 & 1.0694 & 1.1467 \\
\hline
\end{tabular}

Notice that one can make $\Lambda_{\alpha}-T^{*} H^{*} H T$ positive by taking the elements of $\alpha$ arbitrarily large. This proposition implies that this is a poor strategy, as it decreases the convergence rate.

\section{Performance}

To compare the performances of SISTA and ISTA, we conducted a number of experiments. In the first two experiments, we used the same observation signal. To obtain the observation signal, we blurred the signal of length 1024 (zero padded to avoid boundary effects) shown in Fig. 11(a) with a length-30 moving average filter (therefore, neglecting the boundaries, $H$ is a circulant $1024 \times 1024$ matrix) and added Gaussian noise with $\sigma=0.02$. The observed signal is shown in Fig. 11(b). The first two experiments differ by the choice of the frame utilized.

Experiment 1: Using a 6 stage critically sampled DWT with Daubechies filters having four vanishing moments, we ran ISTA for 50000 iterations, thereby obtaining an estimate $x^{*}$ of the solution to problem (2) with $\lambda=0.001$. After computing $\alpha_{1}, \alpha_{2}, \ldots, \alpha_{7}$ using the method in Section V-B (see Table I for these values) we ran SISTA and ISTA for 1000 iterations. For both algorithms, we used $\alpha$ values that are half of that required by MM. In other words, we used $\alpha / 2$ (where $\alpha$ are those values in Table I) for SISTA and $\alpha_{i}=1 / 2$ for ISTA. Fig. 12 shows the logarithm of the RMSE between the iterates of the algorithms

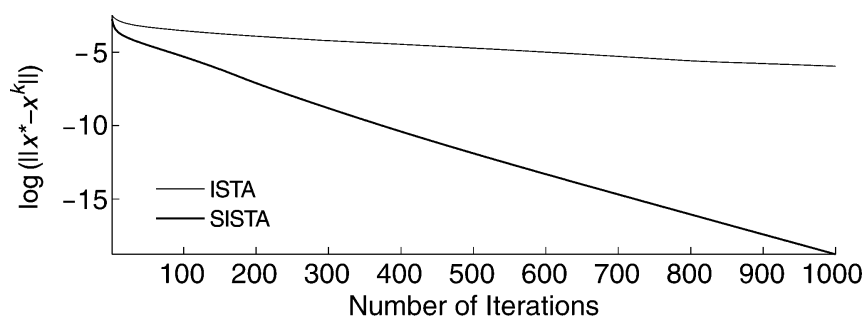

(a)

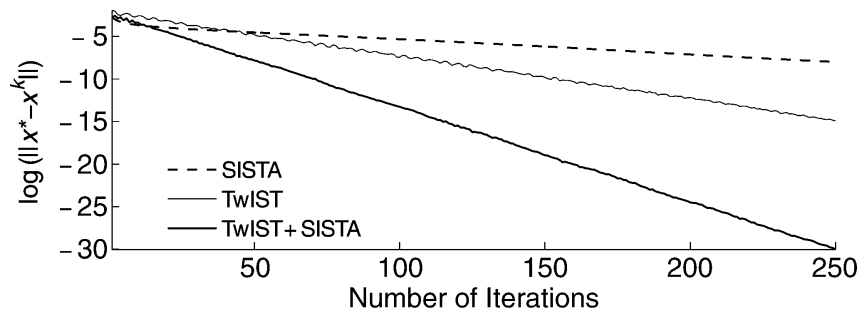

(b)

Fig. 12. $\log \left(\left\|x^{*}-x^{k}\right\|\right)$ versus Number of Iterations for Experiment 1 (Orthonormal Dyadic DWT). (a) Thin curve: ISTA, Thick curve: SISTA. (b) Dashed line: SISTA [from (a)], Thin solid line: TwIST, Thick solid Line: TwIST+SISTA.

and $x^{*}$. According to the definition of the $R$-factor, for high $k$, we expect, noting the orthonormality of the frame that

$$
\log \left(\left\|\mathbf{c}^{k}-\mathbf{c}^{*}\right\|^{1 /(k+b)}\right)=\log \left(\left\|x^{k}-x^{*}\right\|^{1 /(k+b)}\right) \approx \mathcal{R}
$$

i.e.

$$
\log \left\|x^{k}-x^{*}\right\| \approx k \mathcal{R}+b \mathcal{R} .
$$

The constant $b$ is introduced to accomodate for the arbitrariness of the index $k$ ( $x^{10}$ may be equal to $\tilde{x}^{1000}$ for some other sequence). Notice that both curves are fairly linear and the slope of the curve for SISTA is significantly higher in magnitude than that for ISTA, indicating a lower $R$-factor for SISTA.

Even though the speed-up is significant in this case, it is also important to understand where SISTA stands compared to state-of-the-art methods. To gain some insight, we compared the algorithm to TwIST [3], since the definition of 'an iteration' in TwIST is compatible with ISTA and SISTA and therefore it makes sense to track the progress of the algorithms through each iteration (otherwise the comparison has to be somewhat implementation dependent-see [36], for example). If we denote one iteration of ISTA as

$$
c^{k+1}:=\mathcal{M}_{\lambda}\left(c^{k}\right)
$$

then an iteration of TwIST is given by

$$
c^{k+1}:=(1-\gamma) c^{k-1}+(\gamma-\beta) c^{k}+\beta \mathcal{M}_{\lambda}\left(c^{k}\right)
$$

where $\gamma$ and $\beta$ are given or adapted on the run (see [3]). Since the improvement of TwIST stems from combining previous estimates of ISTA, it can readily be adapted to SISTA (by making $\mathcal{M}_{\lambda}(\cdot)$ in (73) subband adaptive). This suggests that SISTA and TwIST are not rival algorithms but can be combined to yield possibly better performance. This indeed turned out to be the case for this example. For the regular TwIST, we hand-tuned the $\gamma$ and $\beta$ parameters for our example and obtained (for $\gamma=$ 


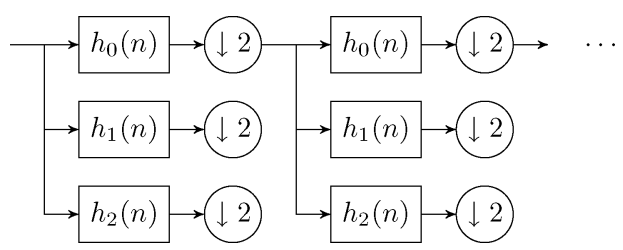

Fig. 13. The double-density DWT is obtained by iterating an oversampled FB. See Experiment 2 for details.

TABLE II

“ $\alpha$ " VeCTOR COMPUTED FOR EXPERIMENT 2. 'LP' STANDS FOR "LOWPASS"

\begin{tabular}{rcrcc}
\hline Subband & 1 & 2 & 3 & 4 \\
\hline LP & - & - & - & 1.3266 \\
I & 0.0118 & 0.0347 & 0.0980 & 0.2325 \\
II & 0.0135 & 0.0397 & 0.1105 & 0.2594 \\
\hline
\end{tabular}

TABLE III

EXAMPLE 1-THE FILTERS FOR THE DOUBLE-DENSITY DWT (SEE FIg. 13) USED IN EXPERIMENT 2

\begin{tabular}{rrrr}
\hline$n$ & $h_{0}(n)$ & \multicolumn{1}{c}{$h_{1}(n)$} & \multicolumn{1}{c}{$h_{2}(n)$} \\
\hline 0 & 0.143015350704421 & -0.442261072417418 & 0 \\
1 & 0.517434399761582 & 0.665607109645973 & -0.446890685359672 \\
2 & 0.639584092002116 & -0.064173111645835 & 0.590890622695870 \\
3 & 0.244299384481069 & -0.117095721362206 & 0.069330257146553 \\
4 & -0.075492661519989 & -0.024412298834887 & -0.123769640942026 \\
5 & -0.054627003056103 & -0.017664905385628 & -0.089560553540724 \\
\hline
\end{tabular}

$1.9, \beta=1.7)$ the log-distance curve shown by the thin line in Fig. 12(b). Compared to the log-distance curve of SISTA, there is a significant improvement. However, if we combine TwIST and SISTA (with hand-tuning $\gamma=1.8$ and $\beta=1$ ) we obtain yet better performance [see the thick line in Fig. 12(b)].

Experiment 2: In the second experiment, we utilized an overcomplete dyadic DWT, namely a double-density DWT (see Fig. 13-also see [29] for more information on this transform) with 4 stages and set $\lambda=0.001$ as in Experiment 1. The filter coefficients are tabulated in Table III. In this setting, we ran ISTA for 50000 iterations to obtain the approximate $\mathbf{c}^{*}$ and $x^{*}=T \mathbf{c}^{*}$, where the latter is shown in Fig. 11(d). Here, since the frame is not orthonormal, we do not have $\|\mathbf{c}\|=\|T \mathbf{c}\|$ and therefore the first equality in (70) does not hold. We can therefore produce two different graphs pertaining to $\left\{\mathbf{c}^{k}\right\}_{k}$ and $\left\{x^{k}\right\}_{k}$. The graph in Fig. 14(a) shows $\log \left(\left\|\mathbf{c}^{k}-\mathbf{c}^{*}\right\|\right)$ versus $k$, and the graph in Fig. 14(b) shows $\log \left(\left\|x^{k}-x^{*}\right\|\right)$ versus $k$. We remark that the asymptotic results presented in Section VI are related to $\log \left(\left\|\mathbf{c}^{k}-\mathbf{c}^{*}\right\|\right)$-see Footnote 5. As in the previous experiment, we computed $\alpha$ using Algorithm 3 (see Table II) and ran SISTA with $\alpha / 2$ and ISTA with $\alpha_{i}=1 / 2$ for 5000 iterations. The log distances are shown in Fig. 14. Observe that even though the convergence rate for SISTA is higher [compare the slopes towards the tail in Fig. 14(a)], the rate of convergence in the 'time-domain' for SISTA and ISTA are approximately equal [compare the slopes towards the tail in Fig. 14(b)]. This observation suggests that, in this example, towards the tail of the sequence $\mathbf{c}^{k}$, most of the action takes place in the null space of $T$, which might not be interesting in an inverse problem setting as discussed in this paper, but which might be interesting if one

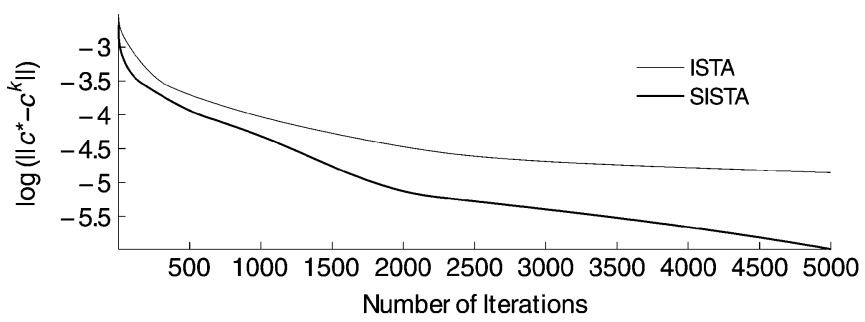

(a)

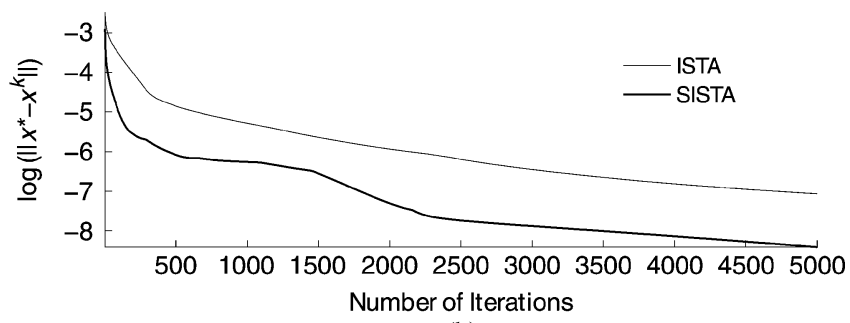

(b)

Fig. 14. Convergence comparison for Experiment 2 (double-density DWT) (a) $\log \left(\left\|\mathbf{c}^{*}-\mathbf{c}^{k}\right\|\right)$ versus Number of Iterations. (b) $\log \left(\left\|x^{*}-x^{k}\right\|\right)$ versus Number of Iterations. In both (a) and (b), Thin curve: ISTA, Thick curve: SISTA.

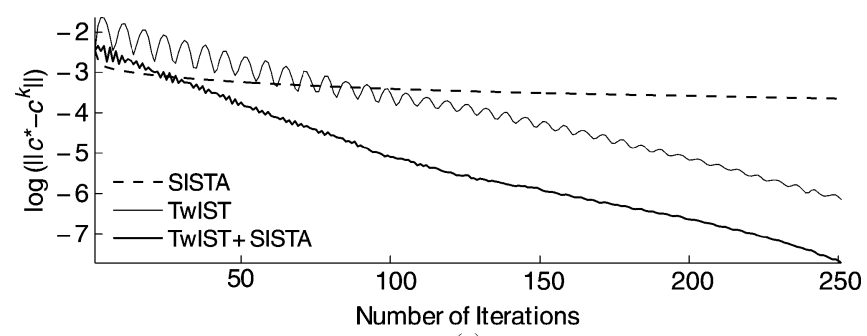

(a)

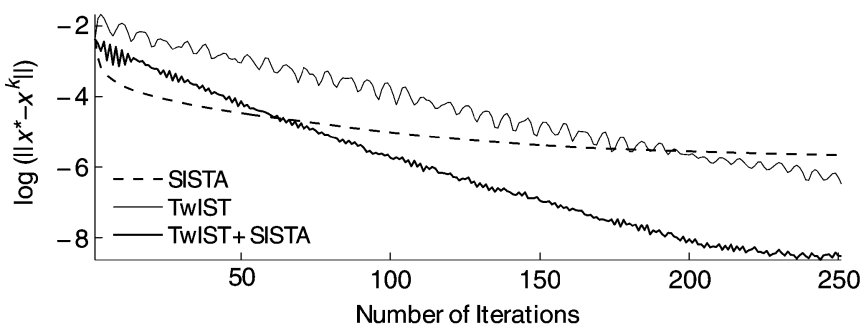

(b)

Fig. 15. Convergence comparison for Experiment 2 (double-density DWT) and TwIST. (a) $\log \left(\left\|\mathbf{c}^{*}-\mathbf{c}^{k}\right\|\right)$ versus Number of Iterations. (b) $\log \left(\left\|x^{*}-x^{k}\right\|\right)$ versus Number of Iterations. In both (a) and (b), Dashed line: SISTA, Thin line: TwIST, Thick line: TwIST+SISTA.

was looking for an approximation of the sparsest representation of the object (e.g., for a coding purpose).

To demonstrate the speed-up with TwIST, we performed another experiment similar to the one described in Experiment 1. In this example too, we hand-tuned the $\gamma$ and $\beta$ parameters to enhance the performance. In particular we set $\gamma=1.96, \beta=1.86$ for TwIST and $\gamma=1.93, \beta=1.48$ for SISTA combined with TwIST. The results are depicted in Fig. 15. Once again we observe that TwIST can be faster than SISTA alone but combining TwIST with SISTA results in yet better performance.

Experiment 3: As a final experiment, we compared the algorithms in a separable 2D deconvolution problem. For the 'cameraman' image (of size $512 \times 512$ ), we normalized the intensity level so that the highest intensity is equal to 1 and added a zero border around the image of size 30 , to avoid boundary effects. 


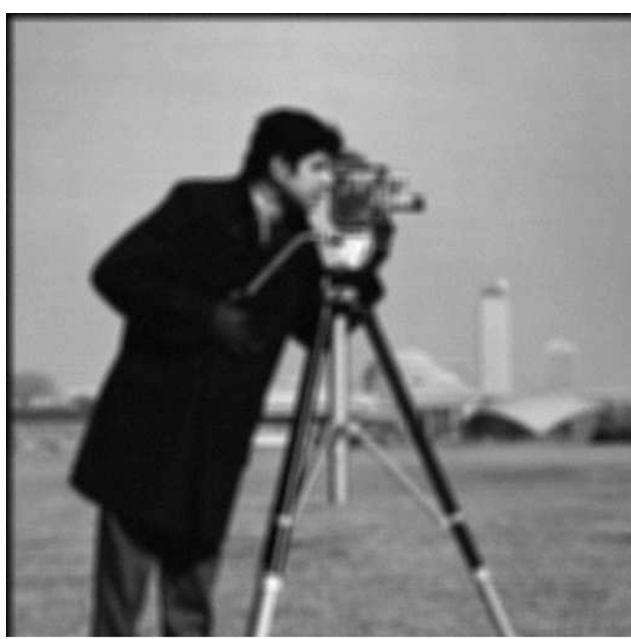

(a)

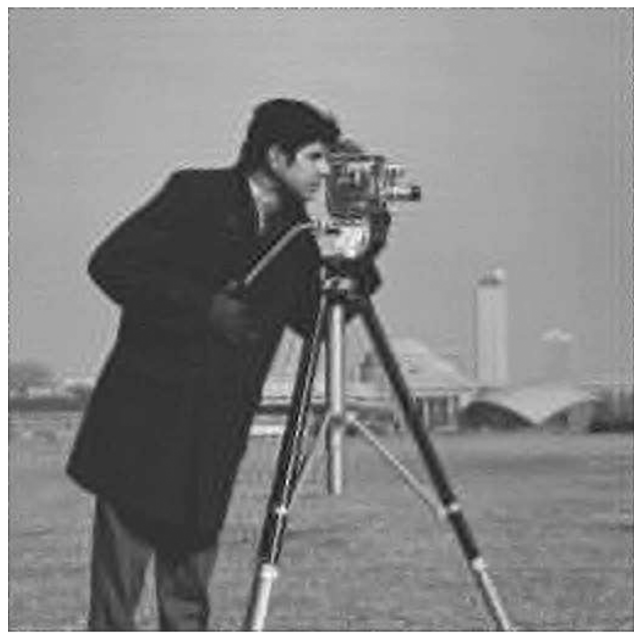

(b)

Fig. 16. (a) Observation. (b) Result of ISTA for 10000 iterations with a 4-stage (13-subband) dyadic separable 2D DWT with Daubechies filters having 4 vanishing moments. In both images, the border is cropped.

TABLE IV

' $\alpha$ ' VeCTOR COMPUTED FOR EXPERIMENT 3. THE $\alpha$ VALUES COMPUTED FOR the HL ChanNel ARe Equal to Those For LH AND ARE Not Listed

\begin{tabular}{rrrrr}
\hline Subband & 1 & 2 & 3 & \multicolumn{1}{c}{4} \\
\hline LL & - & - & - & 1.3430 \\
LH & 0.0399 & 0.1774 & 0.8002 & 1.1577 \\
HH & 0.0009 & 0.0109 & 0.2522 & 0.9919 \\
\hline
\end{tabular}

To obtain the observation signal, we blurred the image with a $9 \times 9$ separable moving average filter (therefore, $H$ may be regarded as a highly structured matrix of size $512 \times 512$ ) and added Gaussian noise with $\sigma=0.02$. This observation signal is shown in Fig. 16(a). Using a 4-stage, separable 2D DWT with Daubechies filters having 4 vanishing moments as the basis, and setting $\lambda=0.005$, we ran the TL algorithm for 10000 iterations and obtained the estimate of the minimizer $x^{*}$ shown in Fig. 16. Proceeding as in the previous experiments, we computed the $\alpha$ vector (see Table IV). The log-distances of ISTA and SISTA are depicted in Fig. 17. As in the 1D experiments, SISTA clearly improves the convergence rate.

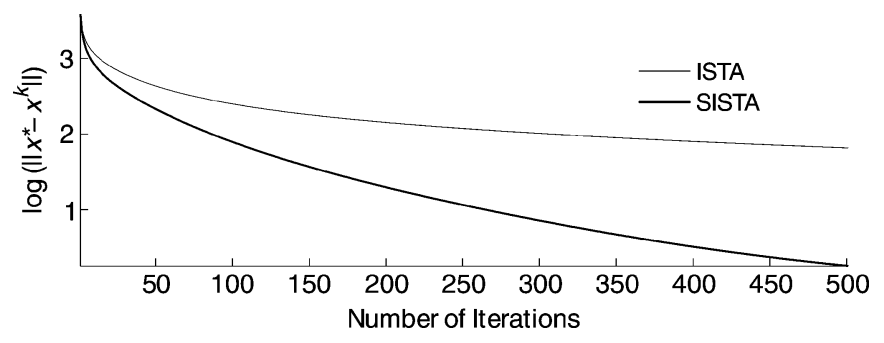

Fig. 17. $\log \left(\left\|x^{*}-x^{k}\right\|\right)$ versus Number of Iterations for Experiment 3 (Orthonormal 2D Dyadic DWT). Thin curve: ISTA, Thick curve: SISTA.

\section{CONCLUSION}

In this paper, we investigated a method to accelerate ISTA, namely SISTA, which was studied in detail by Vonesch and Unser in [33] for the Shannon wavelet basis. SISTA, as well as the original ISTA may be regarded as MM algorithms. In the MM framework, one replaces the cost functional with a more convenient set of surrogate functionals and reaches the optimum by optimizing these surrogate functionals. In particular, ISTA employs a surrogate functional by replacing the data discrepancy term, with another functional that does not discriminate between the subbands. The main idea of SISTA is to replace the data discrepancy term with a subband adaptive functional. In fact, this approach is valid regardless of the regularization term. This subband adaptive functional can be employed in inverse problems that contain a data discrepancy term similar to the one used in this paper. We, therefore, expect this subband adaptive strategy to accelerate similar algorithms where different regularizers (like total variation [27] or $\ell_{1}$ norm of the wavelet analysis coefficients [14]) are used in the problem formulation.

Another interesting aspect of SISTA is that it can readily be used within an acceleration method like TwIST [3] as demonstrated in the Experiments section. We remark that it can also directly be used within the continuation scheme of Hale et al. [17]. Utilizing SISTA alongside these and other schemes like SPARSA [36], FISTA [1] etc. might lead to further acceleration of these algorithms.

\section{ACKNOWLEDGMENT}

The authors would like to thank M. A. T. Figueiredo, Technical University of Lisbon, for bringing [5], [8], and [17] to our attention, M. Unser and C. Vonesch, EPFL, for their comments regarding the comparison to their work and $\mathrm{M}$. Guerquin-Kern, EPFL for comments and discussions. We also would like to thank the anonymous reviewers for their constructive remarks/ suggestions that helped improve the paper.

\section{REFERENCES}

[1] A. Beck and M. Teboulle, "A fast iterative shrinkage-thresholding algorithm for linear inverse problems," SIAM J. Imag. Sci., vol. 1, pp. 183-202, 2009.

[2] M. Bertero and P. Boccacci, Introduction to Inverse Problems in Imaging. New York: Taylor and Francis, 1998.

[3] J. M. Bioucas-Dias and M. A. T. Figueiredo, "A new TwIST: Twostep iterative shrinkage/thresholding algorithms for image restoration," IEEE Trans. Image Process., vol. 16, no. 12, pp. 2992-3003, Dec. 2007.

[4] T. Blumensath and M. E. Davies, "Iterative thresholding for sparse approximations," J. Fourier Anal. Appl., vol. 14, no. 5, pp.629-654, Dec. 2008. 
[5] K. Bredies and D. A. Lorenz, "Linear convergence of iterative softthresholding," J. Fourier Anal. Appl., vol. 14, no. 1, pp. 813-837, Oct. 2008.

[6] K. Bredies, D. A. Lorenz, and P. Maass, "A generalized conditional gradient method and its connection to an iterative shrinkage method," Computat. Optimiz. Appl., vol. 1, no. 1, p. 1-1, Nov. 2007.

[7] C. Chaux, P. L. Combettes, J.-C. Pesquet, and V. R. Wajs, "A variational formulation for frame-based inverse problems," Inverse Problems, vol. 23, no. 4, pp. 1495-1518, Aug. 2007.

[8] P. L. Combettes and V. R. Wajs, "Signal recovery by proximal forwardbackward splitting," SIAM J. Multiscale Model. Simul., vol. 4, no. 4, pp. 1168-1200, Nov. 2005.

[9] L. Şendur and I. W. Selesnick, "Bivariate shrinkage functions for wavelet-based denoising exploiting interscale dependency," IEEE Trans. Signal Process., vol. 50, no. 11, pp. 2744-2756, Jul. 2002.

[10] I. Daubechies, M. Defrise, and C. De Mol, "An iterative thresholding algorithm for linear inverse problems with a sparsity constraint," Commun. Pure Appl. Math., vol. 57, no. 11, pp. 1413-1457, Aug. 2004.

[11] M. Elad, "Why simple shrinkage is still relevant for redundant representations?," IEEE Trans. Inf. Theory, vol. 52, no. 12, pp. 5559-5569, Dec. 2006

[12] M. Elad, B. Matalon, J. Shtok, and M. Zibulevsky, "A wide-angle view at iterated shrinkage algorithms," in Proc. SPIE Wavelets XII, 2007.

[13] M. Elad, B. Matalon, and M. Zibulevsky, "Coordinate and subspace optimization methods for linear least squares with non-quadratic regularization," J. Appl. Comp. Harmon. Anal., vol. 23, pp. 346-367, Nov. 2007.

[14] M. Elad, P. Milanfar, and R. Rubinstein, "Analysis versus synthesis in signal priors," Inverse Problems, vol. 23, no. 3, pp. 947-968, Jun. 2007.

[15] M. A. T. Figueiredo, J. M. Bioucas-Dias, and R. D. Nowak, "Majorization-minimization algorithms for wavelet-based image restoration," IEEE Trans. Image Process., vol. 16, no. 12, pp. 2980-2991, Dec. 2007.

[16] M. A. T. Figueiredo and R. D. Nowak, "An EM algorithm for waveletbased image restoration," IEEE Trans. Image Process., vol. 12, no. 8, pp. 906-916, Aug. 2003.

[17] E. T. Hale, W. Yin, and Y. Zhang, "Fixed-point continuation for $l_{1}$ minimization: Methodology and convergence," SIAM J. Optimiz., vol. 19, no. 3, pp. 1107-1130, Nov. 2008.

[18] R. A. Horn and C. R. Johnson, Matrix Analysis. Cambridge, U.K.: Cambridge Univ. Press, 1990.

[19] D. R. Hunter and K. Lange, "A tutorial on MM algorithms," Amer. Statist., vol. 58, no. 1, pp. 30-37, Feb. 2004.

[20] E. Kreyszig, Introductory Functional Analysis With Applications. New York: Wiley, 1989.

[21] K. Lange, Optimization. New York: Springer, 2004.

[22] K. Lange, D. R. Hunter, and I. Yang, "Optimization transfer using surrogate objective functions," Jr. Comp. Graph. Stat., vol. 9, no. 1, pp. 1-20, Mar. 2000.

[23] D. G. Luenberger, Linear and Nonlinear Programming. Reading, MA: Addison-Wesley, 1984.

[24] C. De Mol and M. Defrise, "A note on wavelet-based inversion methods," in Inverse Problems, Image Analysis and Medical Imaging, M. Z. Nashed and O. Scherzer, Eds. New York: Amer. Math. Soc., 2002.

[25] P. Moulin and J. Liu, "Analysis of multiresolution image denoising schemes using generalized-Gaussian and complexity priors," IEEE Trans. Inf. Theory, vol. 45, no. 3, pp. 909-919, Apr. 1999.

[26] J. M. Ortega and W. C. Rheinboldt, Iterative Solution of Nonlinear Equations in Several Variables. New York: Academic, 1970.

[27] L. Rudin, S. Osher, and E. Fatemi, "Nonlinear total variation based noise removal algorithms," Physica D, vol. 60, no. 1-4, pp. 259-268, Nov. 1992.

[28] S. Sardy, A. G. Bruce, and P. Tseng, "Block coordinate relaxation methods for nonparametric signal denoising with wavelet dictionaries," J. Computat. Graph. Statist., vol. 9, pp. 361-379, 2000.
[29] I. W. Selesnick, "The double density DWT," in Wavelets in Signal and Image Analysis: From Theory to Practice, A. Petrosian and F. G. Meyer, Eds. New York: Kluwer, 2001.

[30] R. G. Shenoy, "Analysis of multirate components and application to multirate filter design," in Proc. IEEE Int. Conf. Acoust., Speech Signal Process. (ICASSP), 1994.

[31] R. G. Shenoy, D. Burnside, and T. W. Parks, "Linear periodic systems and multirate filter design," IEEE Trans. Signal Process., vol. 42, no. 9, pp. 2242-2256, Sep. 1994.

[32] P. P. Vaidyanathan, Multirate Systems and Filter Banks. Englewood Cliffs, NJ: Prentice-Hall, 1992.

[33] C. Vonesch and M. Unser, "A fast thresholded Landweber algorithm for wavelet-regularized multidimensional deconvolution," IEEE Trans. Image Process., vol. 17, no. 4, pp. 539-549, Apr. 2008.

[34] C. Vonesch and M. Unser, "A fast multilevel algorithm for waveletregularized image restoration," IEEE Trans. Image Process., vol. 18, no. 3, pp. 509-523, Mar. 2009.

[35] B. A. Weisburn, T. W. Parks, and R. G. Shenoy, "Error criteria for filter design," in Proc. IEEE Int. Conf. Acoust., Speech Signal Process. (ICASSP), 1994.

[36] S. J. Wright, R. D. Nowak, and M. A. T. Figueiredo, "Sparse reconstruction by separable approximation," IEEE Trans. Signal Process., vol. 57, no. 7, pp. 2479-2493, Jul. 2009.

[37] Y. Zhang and N. Kingsbury, "A Bayesian wavelet-based multidimensional deconvolution with sub-band emphasis," in Proc. IEEE Int. Conf. Eng. Med. Biol. Soc. (EMBS), 2008.

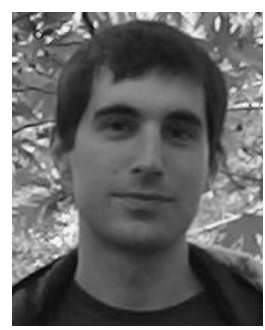

İlker Bayram received the B.Sc. and M.Sc. degrees in electrical and electronics engineering from Middle East Technical University (METU), Ankara, Turkey, and the Ph.D. degree in electrical and computer engineering from Polytechnic Institute of New York University, Brooklyn, in 2002, 2004, and 2009, respectively.

He is currently a Postdoctoral Researcher with the Biomedical Imaging Group, Swiss Federal Institute of Technology, Lausanne (EPFL). His research interests are in wavelets, filter banks, and their applications in inverse problems.

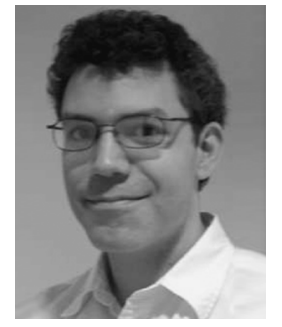

Ivan W. Selesnick received the B.S., M.E.E., and $\mathrm{Ph} . \mathrm{D}$. degrees in electrical and computer engineering in 1990, 1991, and 1996, respectively, from Rice University, Houston, TX.

In 1997, he was a visiting professor with the University of Erlangen-Nürnberg, Germany. Since 1997, he has been with the Department of Electrical and Computer Engineering, Polytechnic Institute of New York University (formerly Polytechnic University), Brooklyn, where he is currently an Associate Professor. His current research interests are in the area of digital signal processing and wavelet-based signal processing.

Dr. Selesnick received a DARPA-NDSEG fellowship in 1991. His Ph.D. dissertation received the Budd Award for Best Engineering Thesis at Rice University in 1996, and an award from the Rice-TMC chapter of Sigma Xi. He received an Alexander von Humboldt Fellowship in 1997 and a National Science Foundation Career award in 1999. In 2003, he received a Jacobs Excellence in Education Award from Polytechnic University. He has been an Associate Editor of the IEEE TRANSACTIONS ON IMAGE PROCESSING and the IEEE SigNAL PROCESSING LETTERS. He is currently an Associate Editor of the IEEE TRANSACTIONS ON SIGNAL PROCESSING. 